\title{
Liquid-Assisted Grinding Accelerating: Suzuki-Miyaura Reaction of Aryl Chlorides under High-Speed Ball-Milling Conditions
}

\author{
Zhi-Jiang Jiang, ${ }^{\text {a } Z h e n-H u a ~ L i, ~}{ }^{*, b}$ Jing-Bo Yu, ${ }^{a}$ Wei-Ke Su*,
}

${ }^{a}$ National Engineering Research Center for Process Development of Active Pharmaceutical Ingredients, Collaborative Innovation Center of Yangtze River Delta Region Green Pharmaceuticals, Zhejiang University of Technology, Hangzhou 310014, P. R. China

${ }^{b}$ Key Laboratory for Green Pharmaceutical Technologies and Related Equipment of Ministry of Education, College of Pharmaceutical Sciences, Zhejiang University of Technology, Hangzhou 310014, P. R. China

\section{Table of Contents}

1. Optimization studies

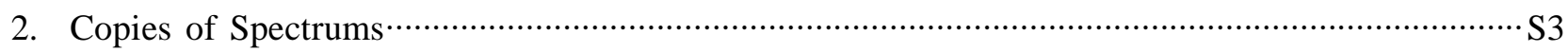

\section{Optimization Studies}

Table S1. Optimization of mechanochemistry process parameters using Davephos as ligand. ${ }^{a}$

\begin{tabular}{|c|c|c|c|c|c|}
\hline no. & frequency $(\mathrm{Hz})$ & time (min) & $\% \varphi^{b}$ & grinding (mg) & $\%$ yield $^{c}$ \\
\hline 1 & 30 & 60 & 5.7 & silica gel (200) & 35 \\
\hline 2 & 25 & 60 & 5.7 & silica gel (200) & 32 \\
\hline 3 & 20 & 60 & 5.7 & silica gel (200) & 30 \\
\hline 4 & 30 & 90 & 5.7 & silica gel (200) & 37 \\
\hline 5 & 30 & 120 & 5.7 & silica gel (200) & 40 \\
\hline 6 & 30 & 60 & $11.5^{d}$ & silica gel (200) & 48 \\
\hline 7 & 30 & 60 & $17.2^{e}$ & silica gel (200) & 45 \\
\hline 8 & 30 & 60 & $11.5^{d}$ & $\mathrm{NaCl}(500)$ & 47 \\
\hline 9 & 30 & 60 & $11.5^{d}$ & $\mathrm{Na}_{2} \mathrm{SO}_{4}(500)$ & 43 \\
\hline 10 & 30 & 60 & $11.5^{d}$ & silica gel (100) & 50 \\
\hline 11 & 30 & 60 & $11.5^{d}$ & silica gel (300) & 45 \\
\hline 12 & 30 & 60 & $11.5^{d}$ & - & 55 \\
\hline
\end{tabular}

${ }^{a}$ Reaction conditions unless specified otherwise: 1a $(0.5 \mathrm{mmol}), 2 \mathrm{a}(0.6 \mathrm{mmol}), \mathrm{Pd}(\mathrm{OAc})_{2}(10 \mathrm{~mol} \%)$, Davephos $(10 \mathrm{~mol} \%), \mathrm{K}_{2} \mathrm{CO}_{3}$ (2.0 equiv.) and Silica gel $(200 \mathrm{mg})$ were placed in a $25 \mathrm{~mL}$ stainless-steel vessel with one stainless-steel ball $(\varnothing=1.4 \mathrm{~cm}) .{ }^{b} \varphi$ represent the filling degree of grinding balls. ${ }^{c}$ Yield based on 1a, average of three runs. ${ }^{d}$ Two stainless-steel balls $(\varnothing=1.4 \mathrm{~cm})$ were used. ${ }^{e}$ Three stainless-steel balls $(\varnothing=1.4 \mathrm{~cm})$ were used. 
Table S2. Optimization of reagent system using $\mathrm{PCy}_{3}$ as ligand. ${ }^{a}$

\begin{tabular}{ccccc}
\hline no. & catalyst (mol\%) & Base (equiv.) & time/min & \% yield $^{\boldsymbol{b}}$ \\
\hline 1 & $\mathrm{Pd}(\mathrm{OAc})_{2}(10)$ & $\mathrm{K}_{2} \mathrm{CO}_{3}(2.0)$ & 120 & 21 \\
2 & $\mathrm{PdCl}_{2}(10)$ & $\mathrm{K}_{2} \mathrm{CO}_{3}(2.0)$ & 120 & 17 \\
3 & $\mathrm{Pd}(\mathrm{TFA})_{2}(10)$ & $\mathrm{K}_{2} \mathrm{CO}_{3}(2.0)$ & 120 & 20 \\
4 & $\mathrm{Pd}(\mathrm{OAc})_{2}(10)$ & $\mathrm{NaOH}(2.0)$ & 120 & 15 \\
5 & $\mathrm{Pd}(\mathrm{OAc})_{2}(10)$ & $\mathrm{KOH}(2.0)$ & 120 & 16 \\
6 & $\mathrm{Pd}(\mathrm{OAc})_{2}(10)$ & $\mathrm{KF}(2.0)$ & 120 & 17 \\
7 & $\mathrm{Pd}(\mathrm{OAc})_{2}(10)$ & $\mathrm{CsF}(2.0)$ & 120 & 17 \\
8 & $\mathrm{Pd}(\mathrm{OAc})_{2}(10)$ & $\mathrm{K}_{2} \mathrm{CO}_{3}(3.0)$ & 120 & 30 \\
9 & $\mathrm{Pd}(\mathrm{OAc})_{2}(10)$ & $\mathrm{K}_{2} \mathrm{CO}_{3}(4.0)$ & 120 & 32 \\
10 & $\mathrm{Pd}(\mathrm{OAc})_{2}(10)$ & $\mathrm{K}_{2} \mathrm{CO}_{3}(5.0)$ & 120 & 41 \\
11 & $\mathrm{Pd}(\mathrm{OAc})_{2}(10)$ & $\mathrm{K}_{2} \mathrm{CO}_{3}(5.0)$ & 60 & 38 \\
12 & $\mathrm{Pd}(\mathrm{OAc})_{2}(5)$ & $\mathrm{K}_{2} \mathrm{CO}_{3}(5.0)$ & 60 & 38 \\
\hline
\end{tabular}

${ }^{a}$ Reaction conditions unless specified otherwise: 1a $(0.5 \mathrm{mmol})$, $\mathbf{2 a}(0.6 \mathrm{mmol})$, catalyst, $\mathrm{PCy}_{3} \cdot \mathrm{HBF}_{4}$ ( 2 folds of catalyst used), and base were placed in a $25 \mathrm{~mL}$ stainless-steel vessel with two stainless-steel balls $(\varnothing=1.4 \mathrm{~cm})$. Ball milling at $30 \mathrm{~Hz} .{ }^{b}$ Yield based on $\mathbf{1}$, average of 3 runs.

Table S3. Further optimization of $\mathrm{PCy}_{3} / \mathrm{MeOH}$ system. ${ }^{a}$

\begin{tabular}{cccccc}
\hline no. & Pd(OAc) $\mathbf{2}(\mathbf{m o l} \%)$ & $\mathbf{K}_{\mathbf{2}} \mathbf{C O}_{\mathbf{3}}(\mathbf{e q u i v} \mathbf{)}$ & $\mathbf{M e O H}(\boldsymbol{\eta})$ & time(min) & \% yield $^{\boldsymbol{b}}$ \\
\hline 1 & 5 & 5.0 & 0.045 & 60 & 97 \\
2 & 3 & 5.0 & 0.045 & 60 & 90 \\
3 & 2 & 5.0 & 0.045 & 60 & 85 \\
4 & 1 & 5.0 & 0.045 & 60 & $82^{c}(87)^{d}$ \\
5 & 0.5 & 5.0 & 0.045 & 60 & $75^{c}(77)^{d}$ \\
6 & 2 & 3.0 & 0.045 & 60 & $75^{c}(82)^{d}$ \\
7 & 2 & 5.0 & 0.050 & 60 & 85 \\
8 & 2 & 5.0 & 0.040 & 60 & 79 \\
9 & 2 & 5.0 & 0.035 & 60 & 73 \\
10 & 2 & 5.0 & 0.040 & 99 & 84 \\
10 & 2 & 5.0 & 0.040 & 120 & 89 \\
11 & 2 & 5.0 & 0.045 & 99 & 97 \\
\hline
\end{tabular}

${ }^{a}$ Reaction conditions unless specified otherwise: 1a $(1.0 \mathrm{mmol}), \mathbf{2 a}(1.2 \mathrm{mmol}), \mathrm{Pd}(\mathrm{OAc})_{2}, \mathrm{PCy}_{3} \cdot \mathrm{HBF}_{4}$ (2 folds of $\mathrm{Pd}(\mathrm{OAc})_{2}$ used), $\mathrm{K}_{2} \mathrm{CO}_{3}$, and $\mathrm{MeOH}$ were placed in a $25 \mathrm{~mL}$ stainless-steel vessel with two stainless-steel balls $(\varnothing=$ $1.4 \mathrm{~cm}$ ). Ball milling at $30 \mathrm{~Hz} .{ }^{b}$ Yield based on 1a, average of three runs. ${ }^{c}$ Poor reproducibility. ${ }^{d}$ Best yield. 
2. Copies of Spectrums

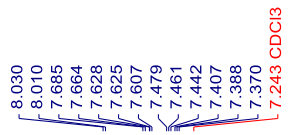

势)

홍

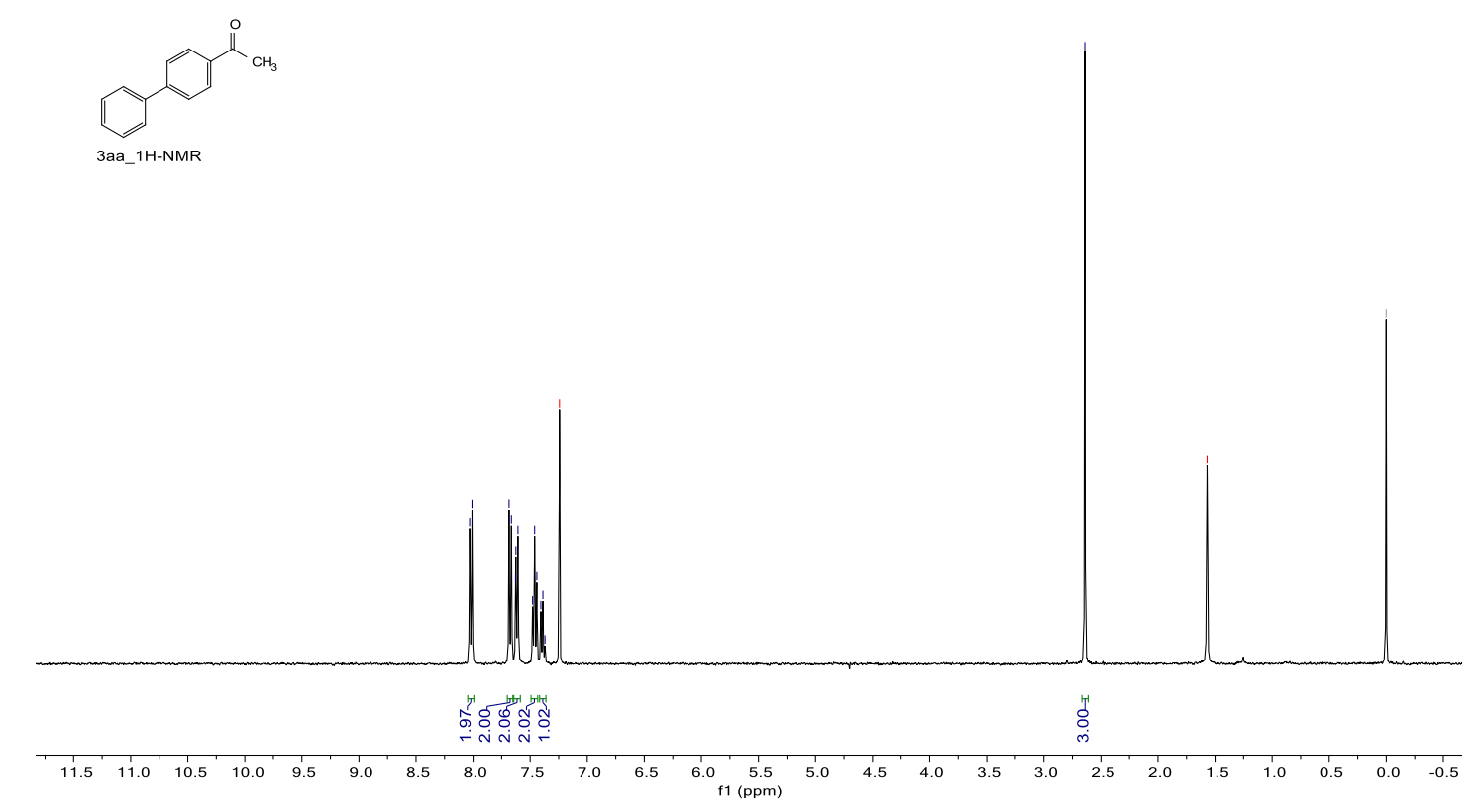

1

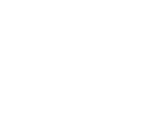

(n)
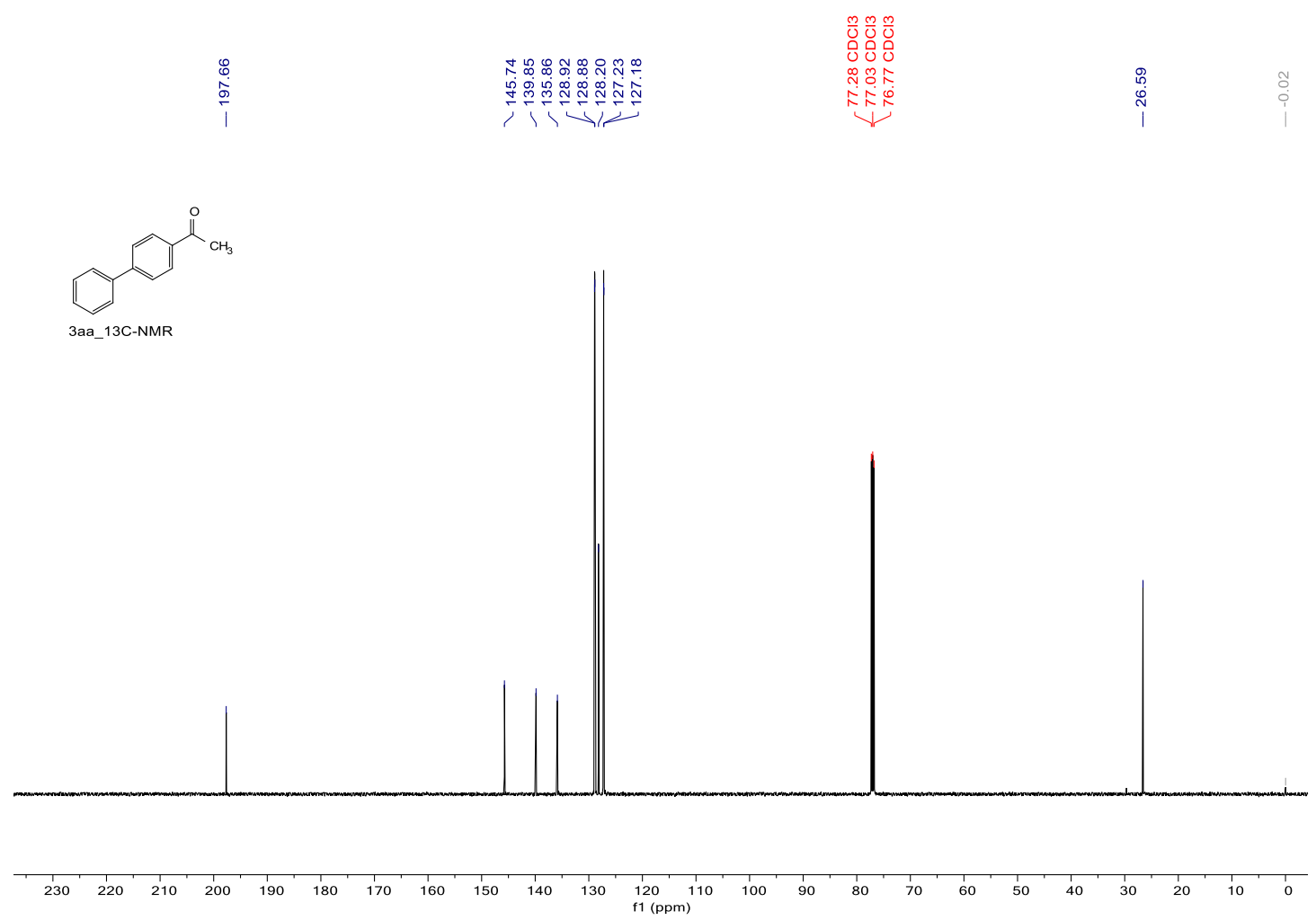

S3 


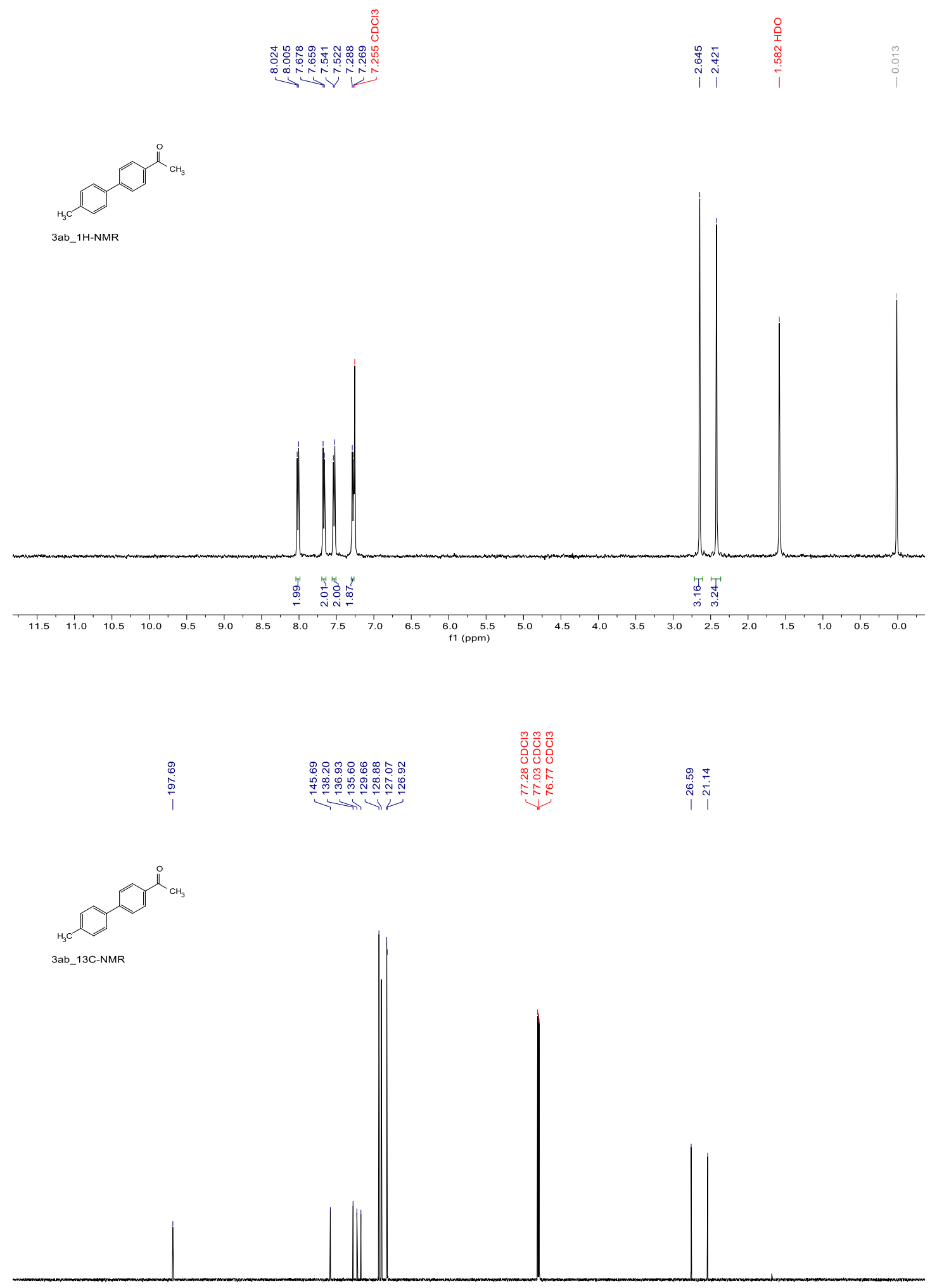

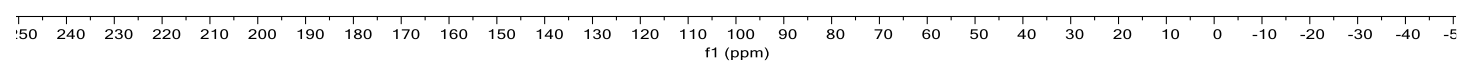




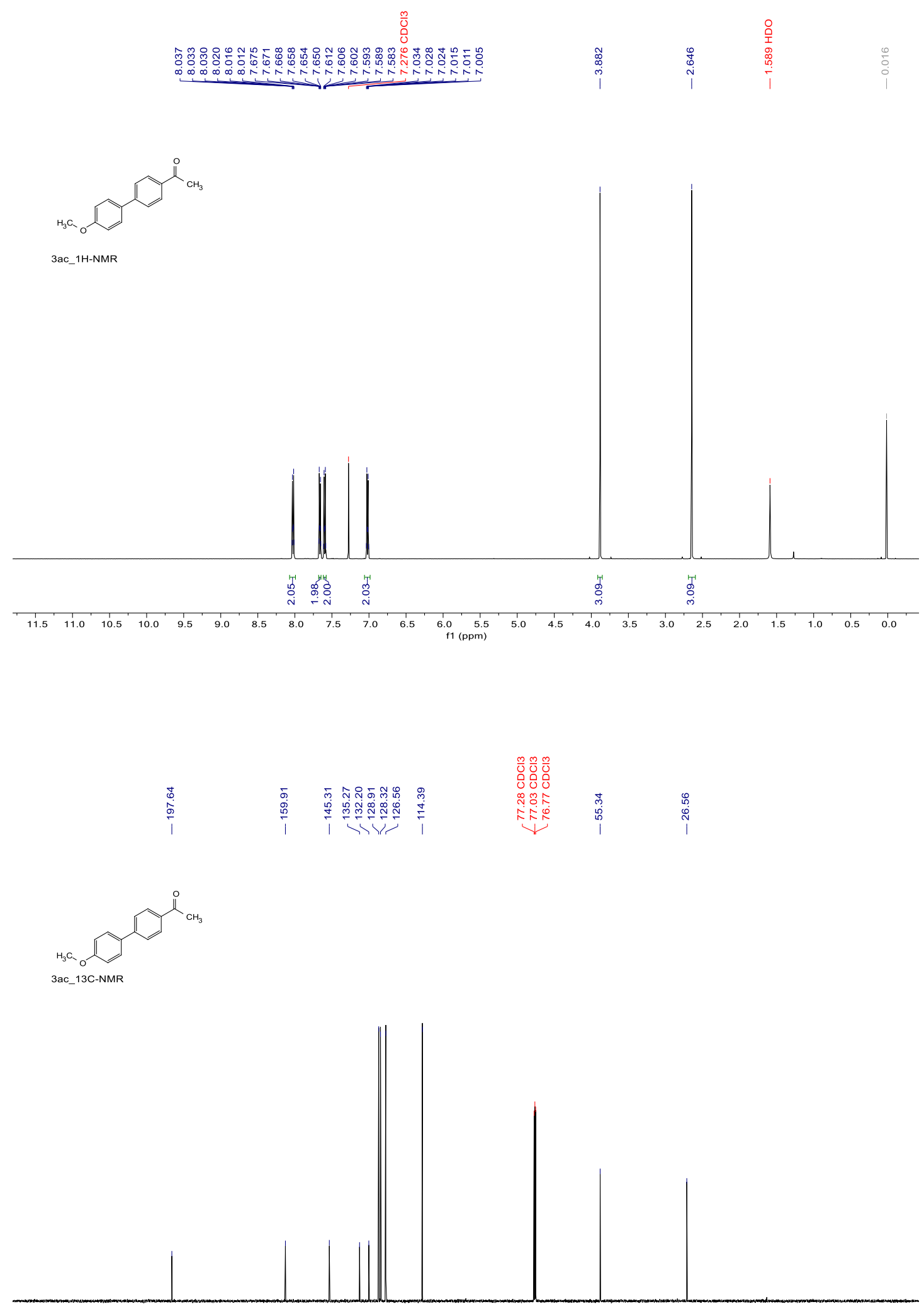

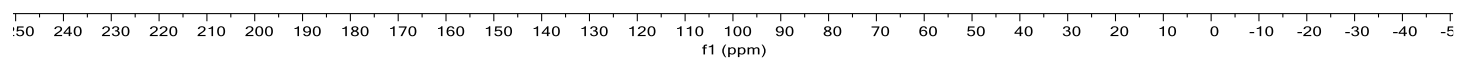




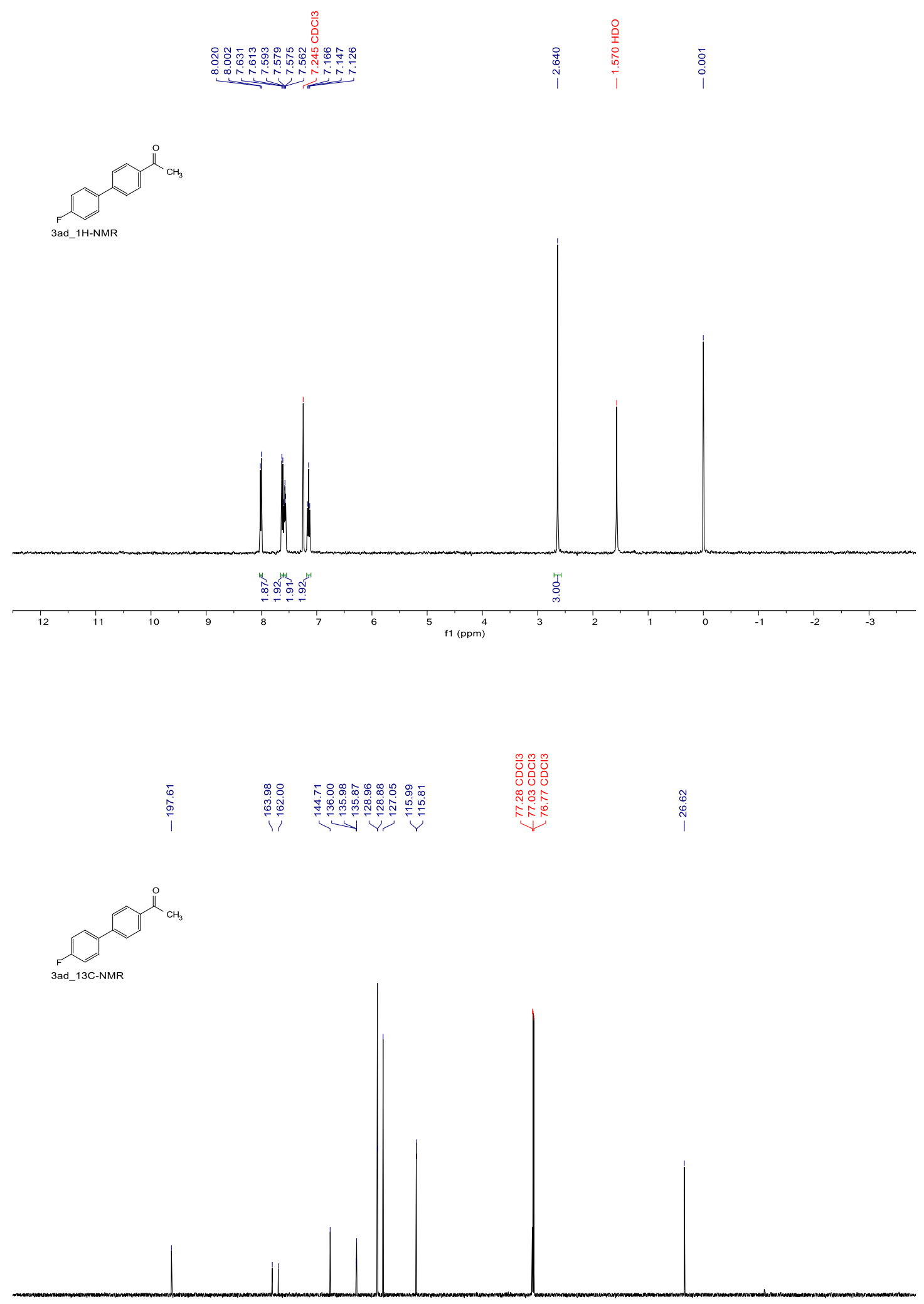

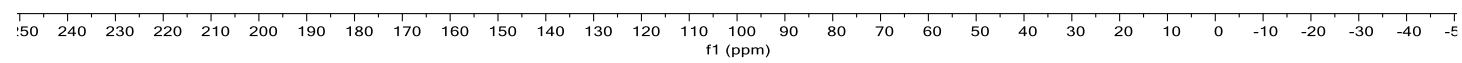



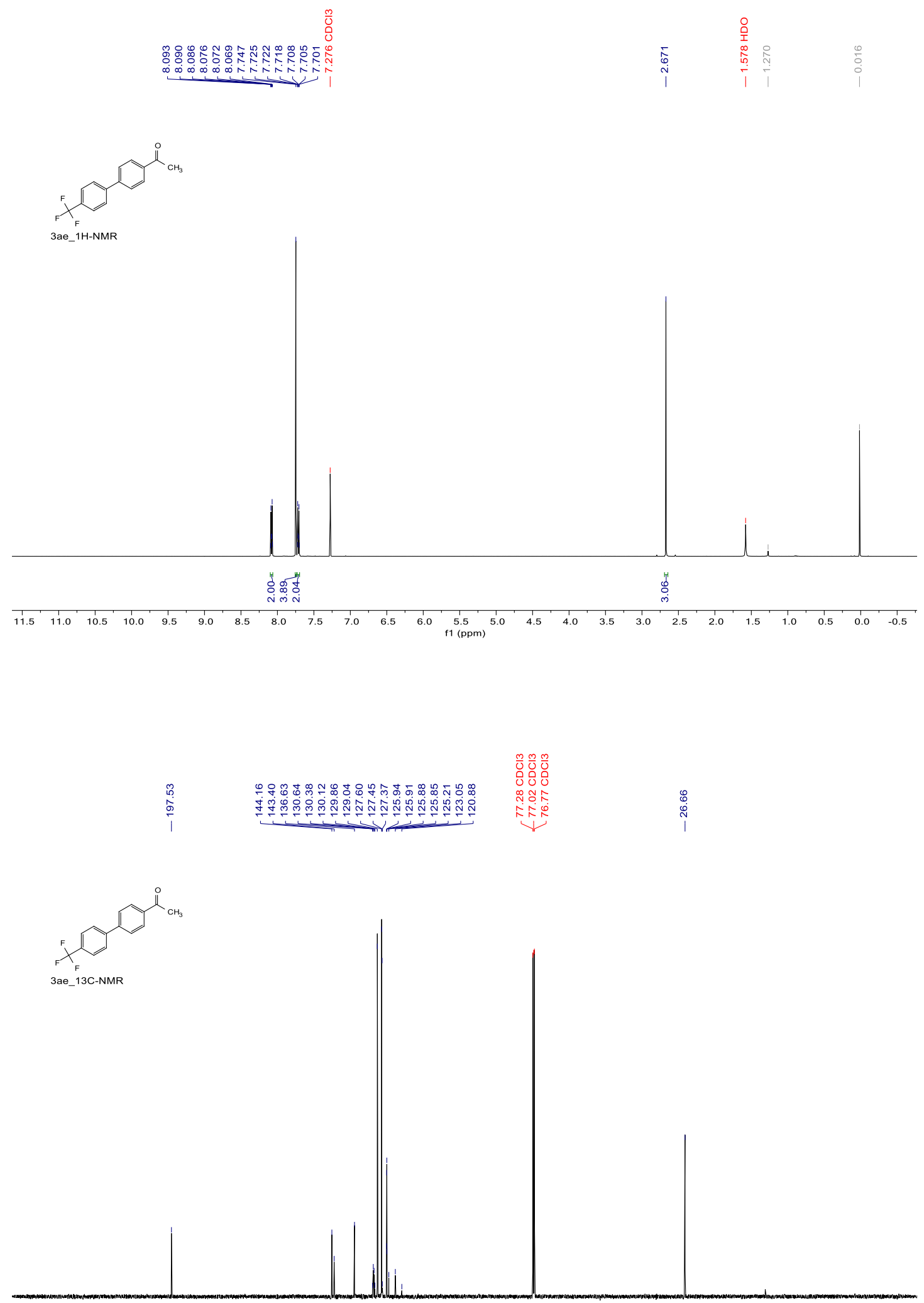

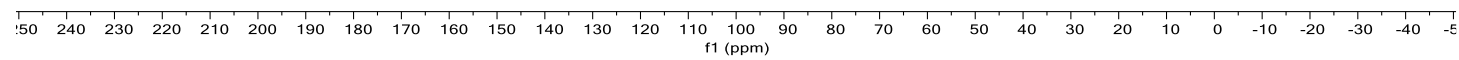



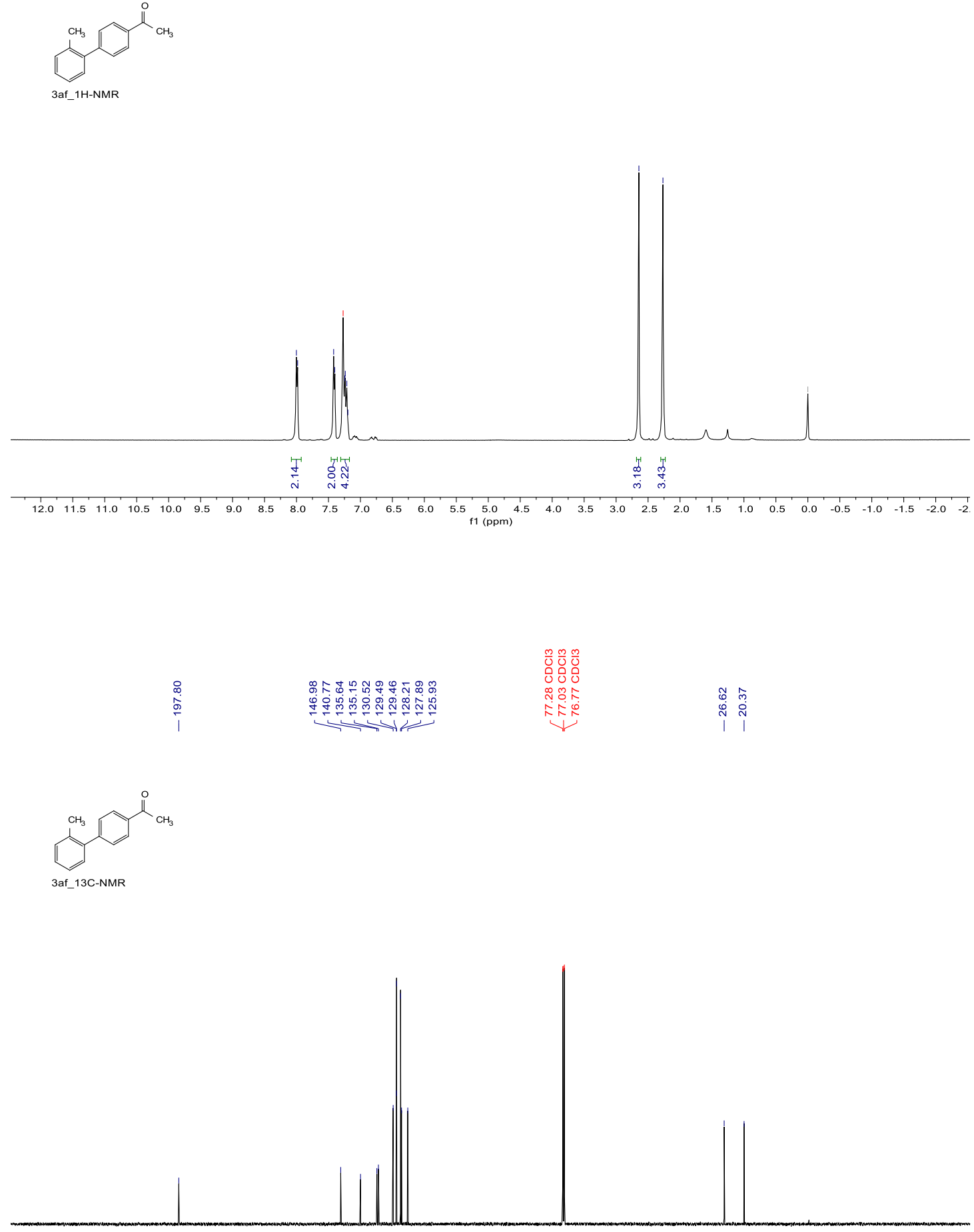

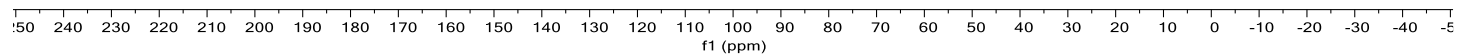



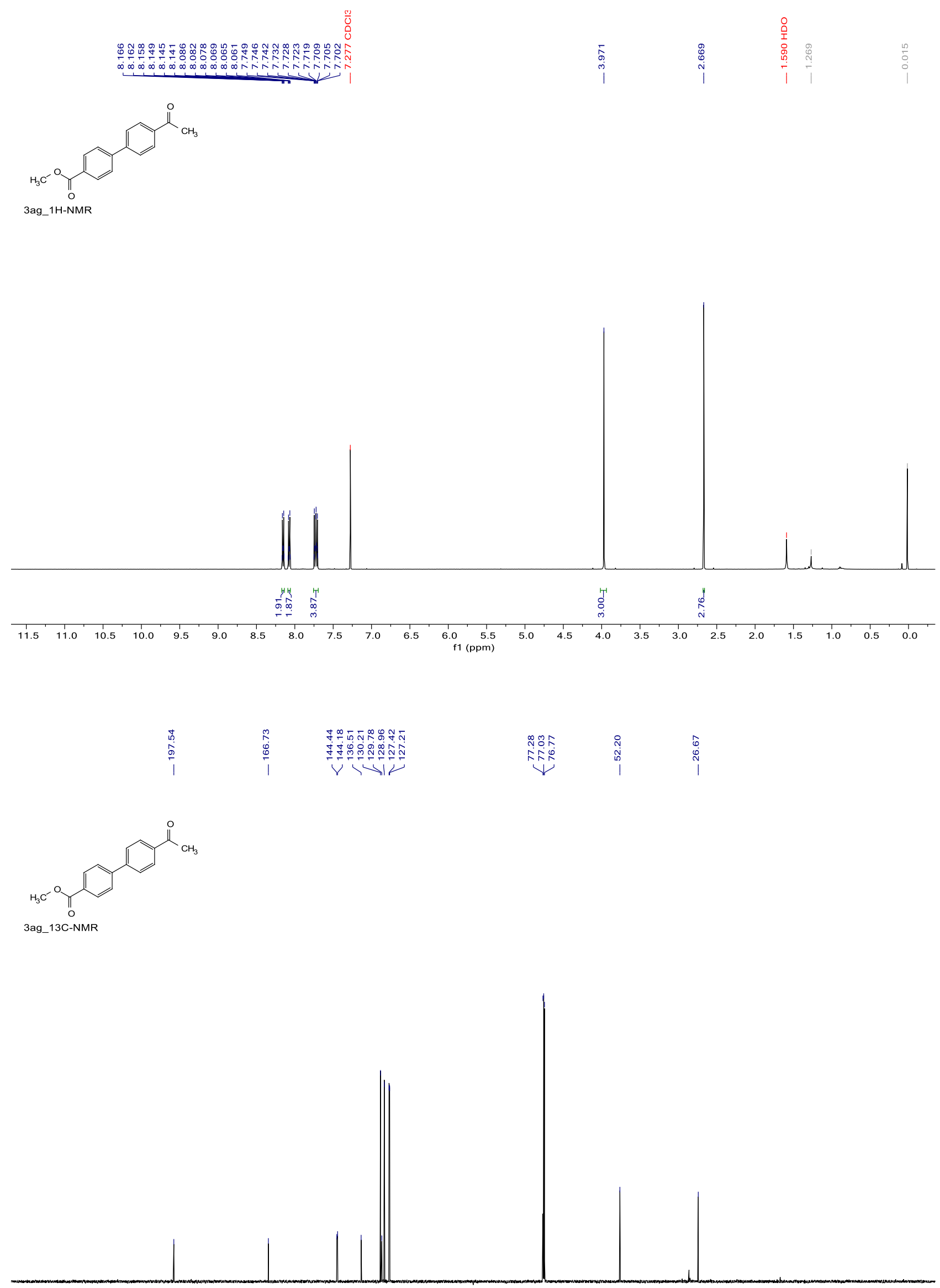

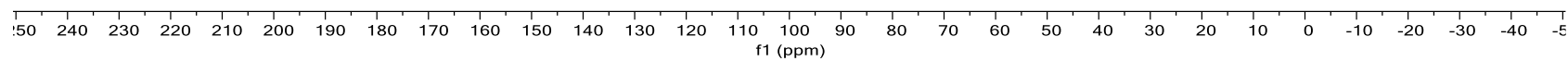


<smiles>CC(=O)c1ccc(-c2ccc([N+](=O)[O-])cc2)cc1</smiles>

3ah_1H-NMR
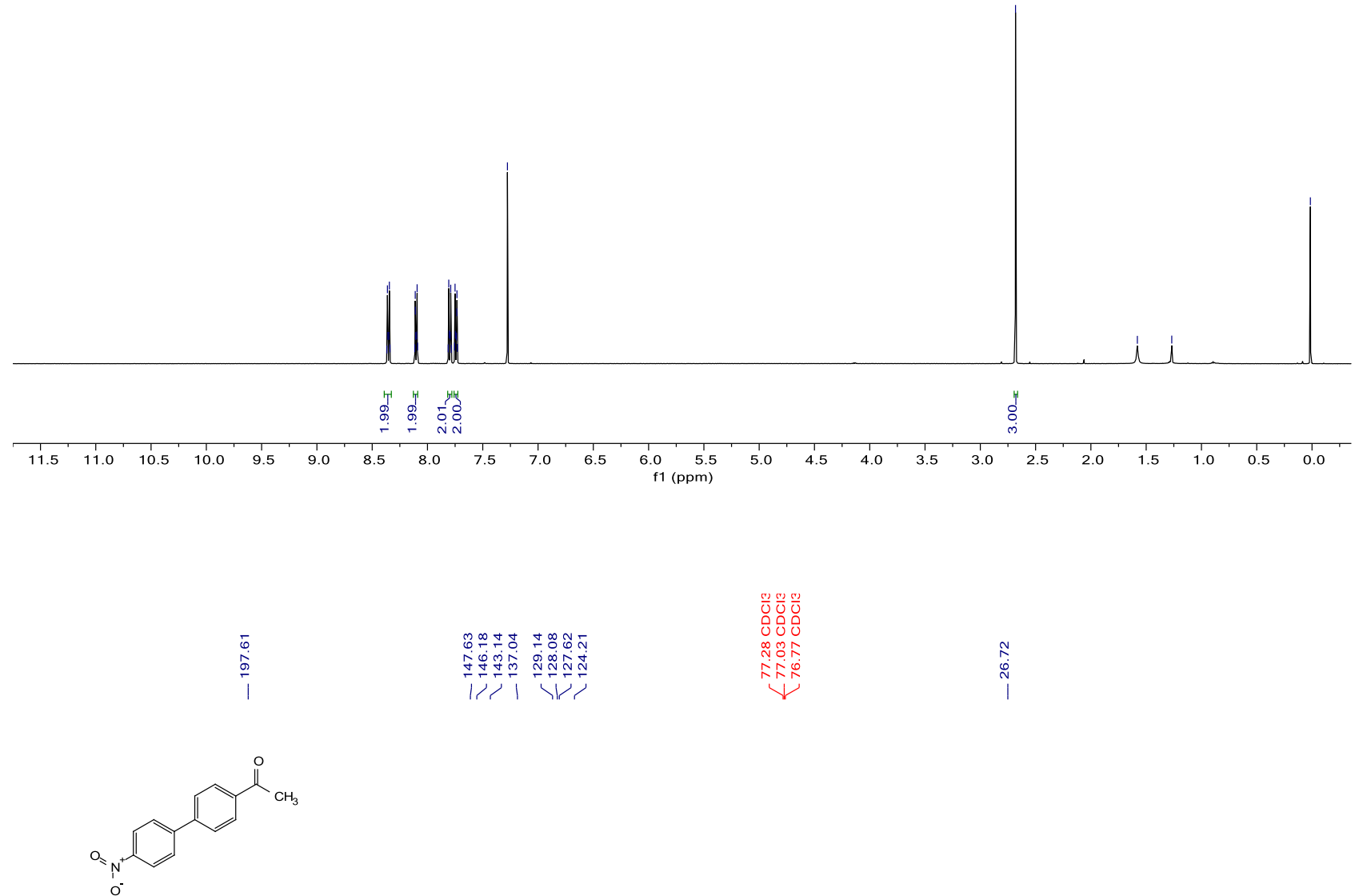

3ah_13C-NMR

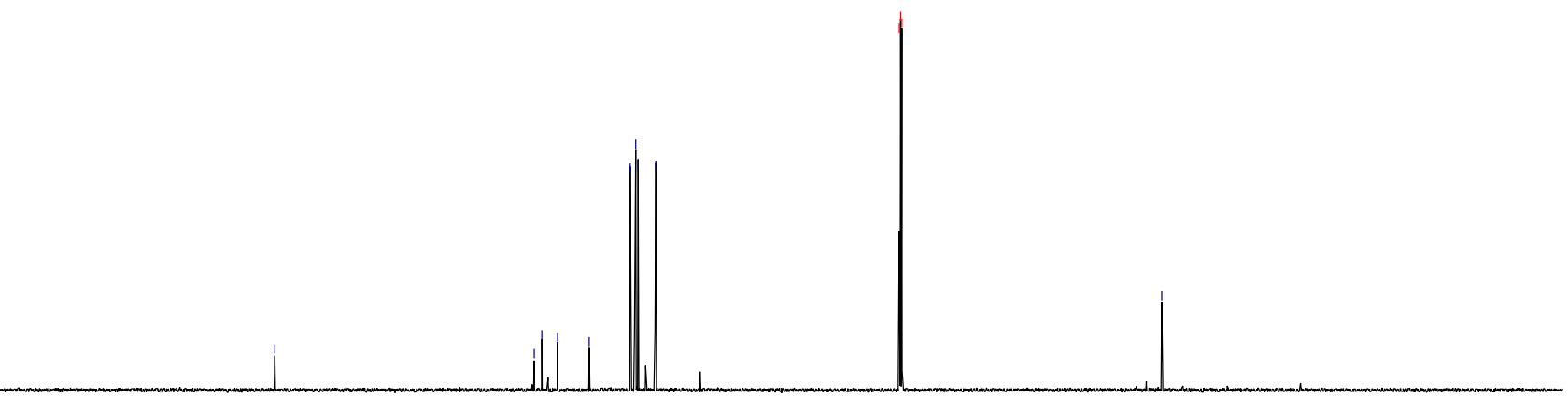

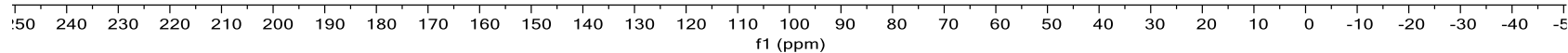



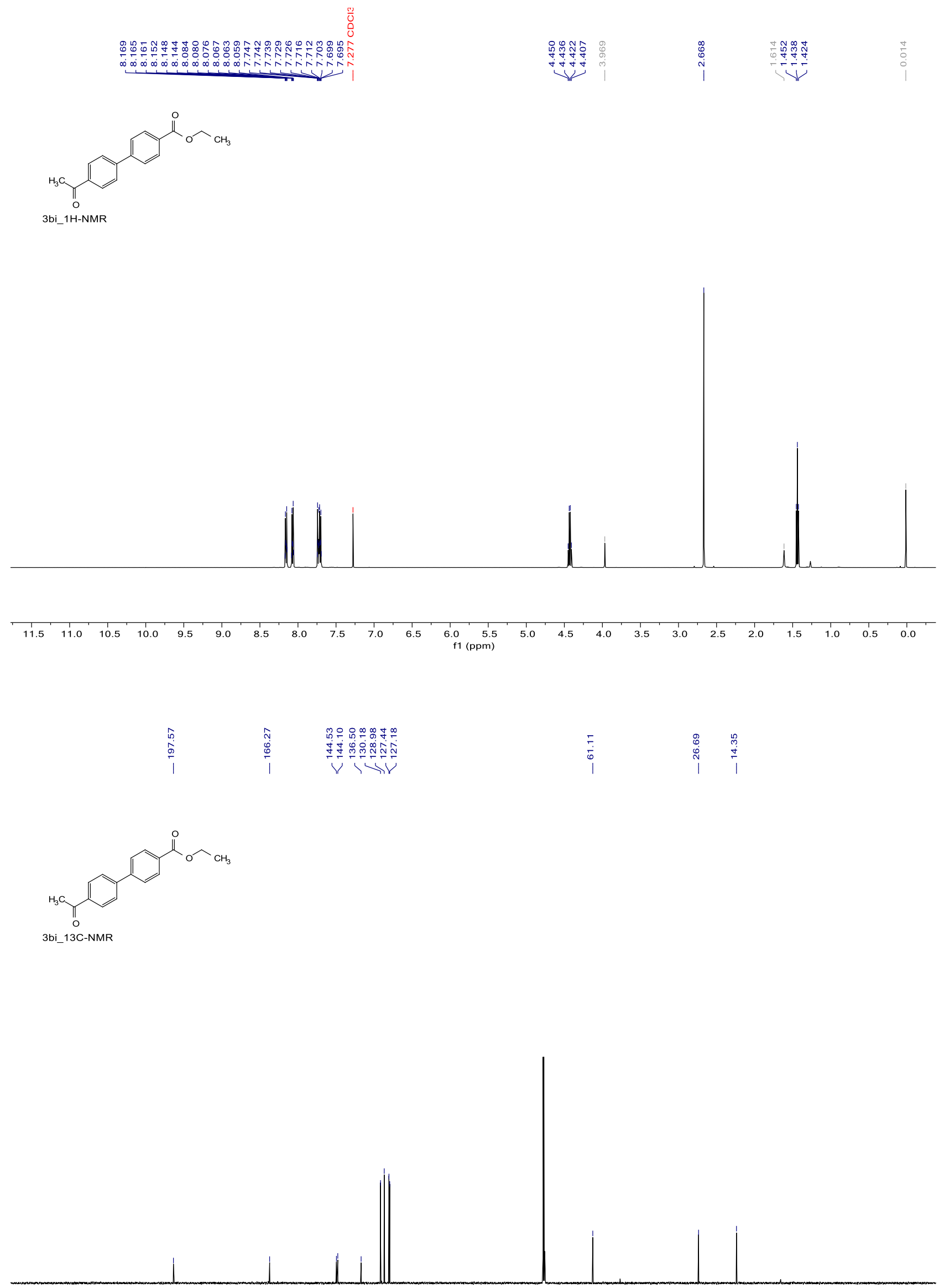

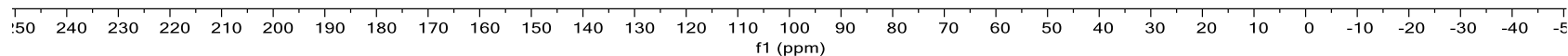



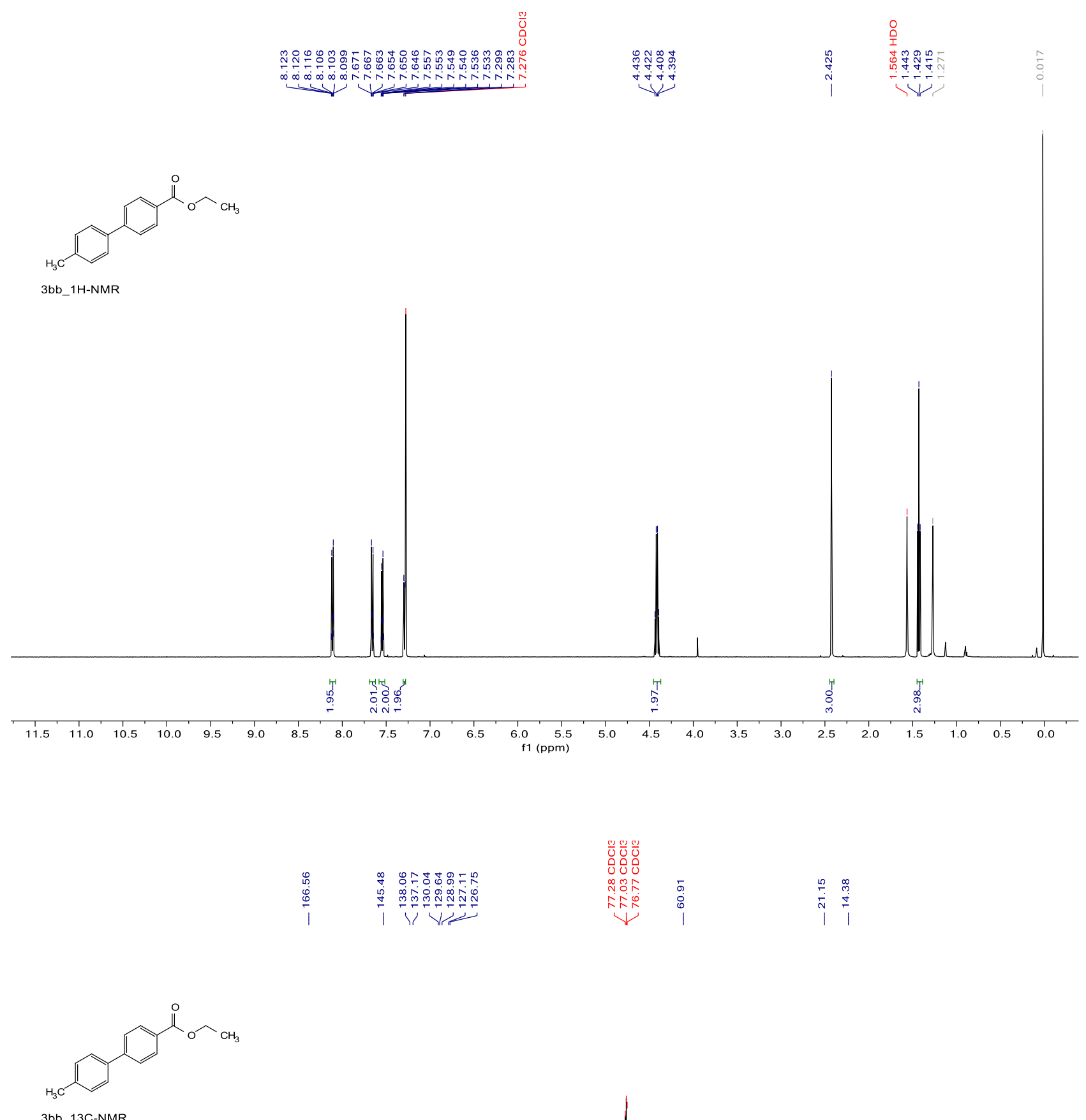

3bb_13C-NMR

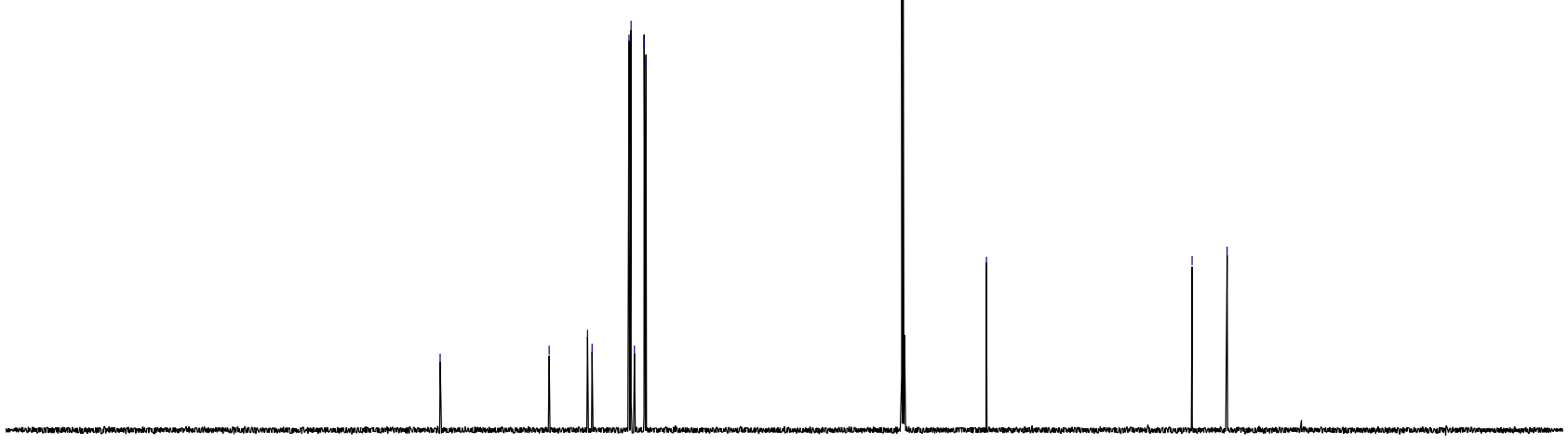

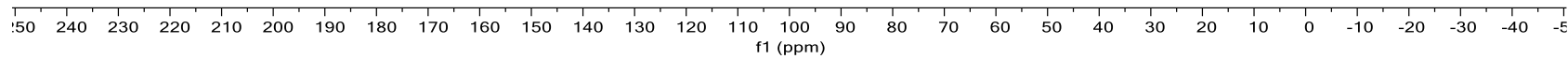




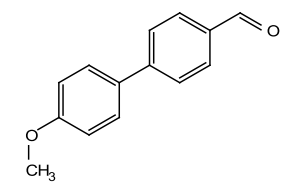

3cc_1H-NMR
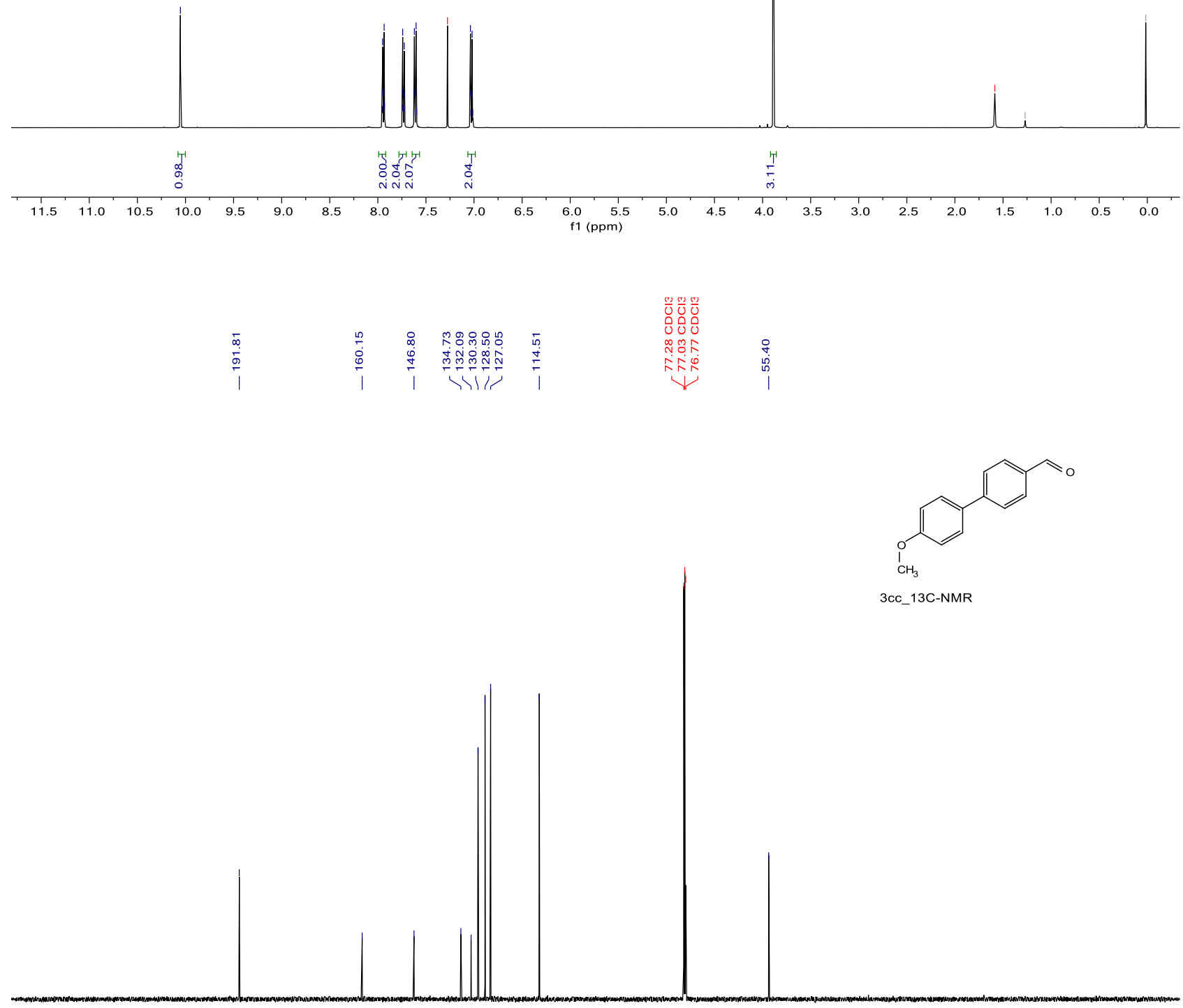

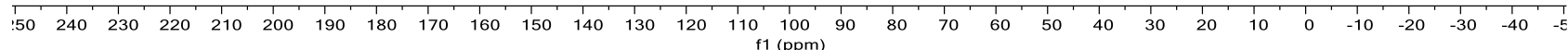



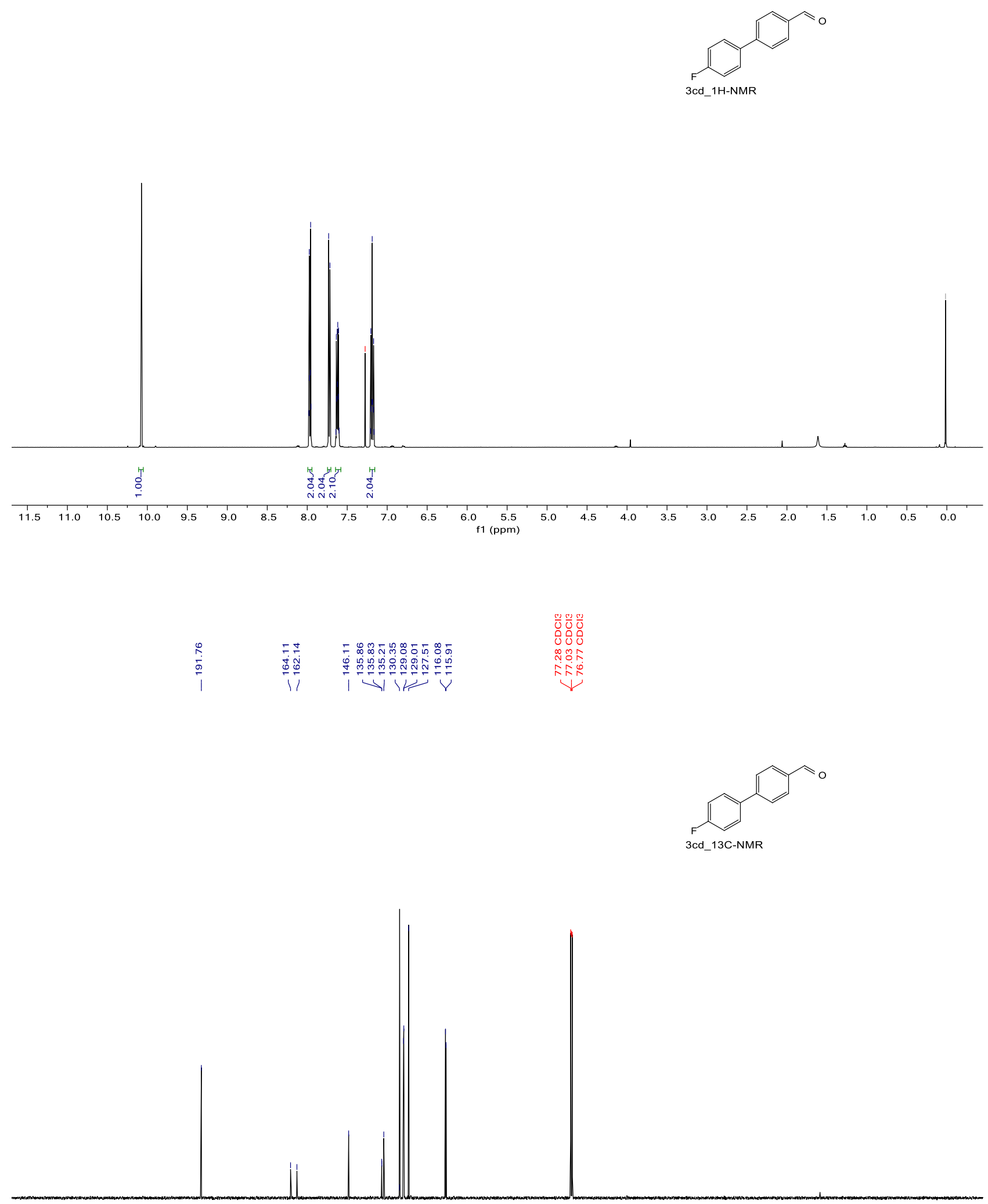

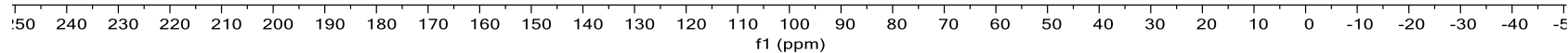




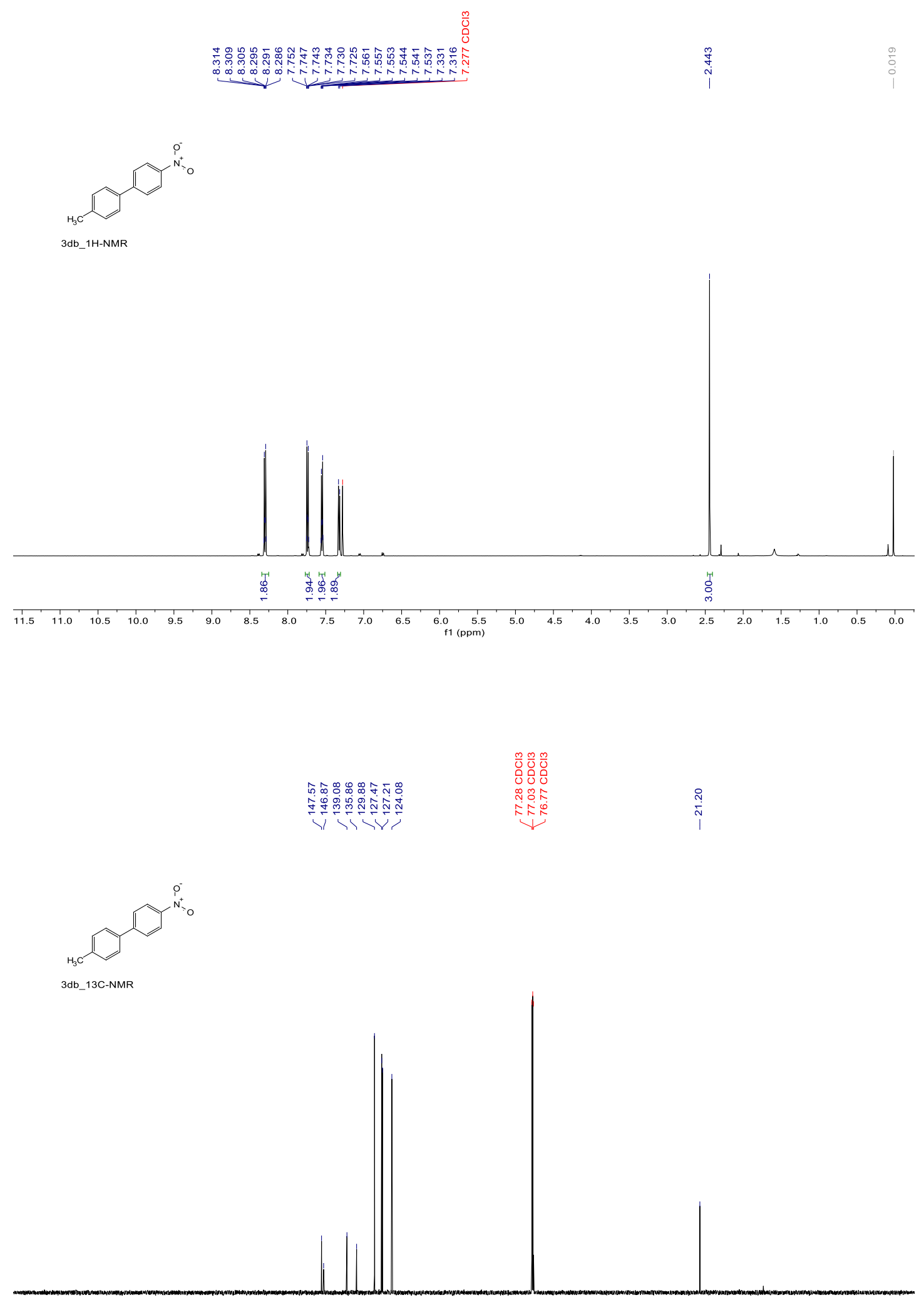

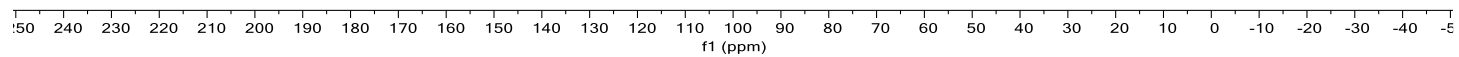




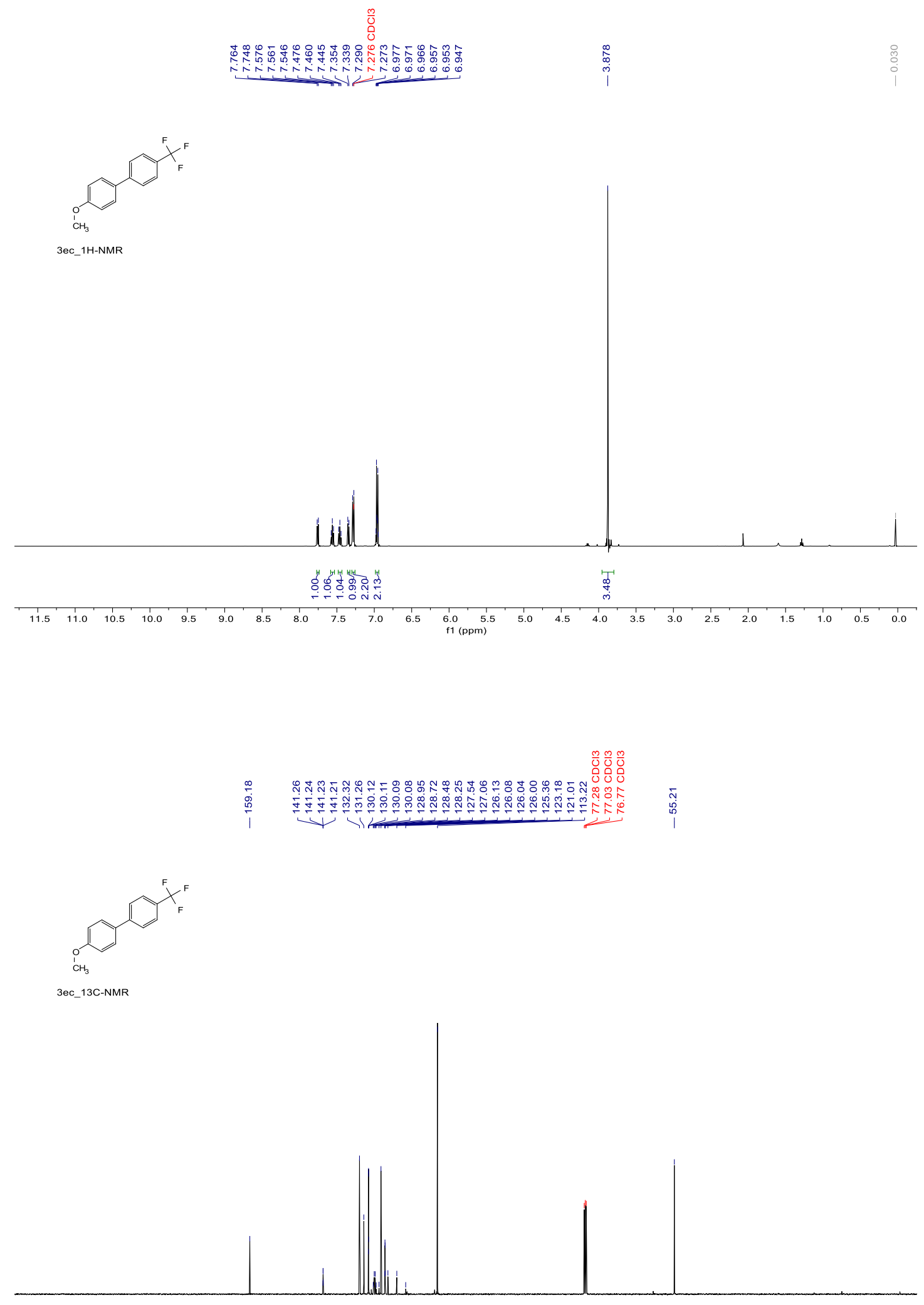

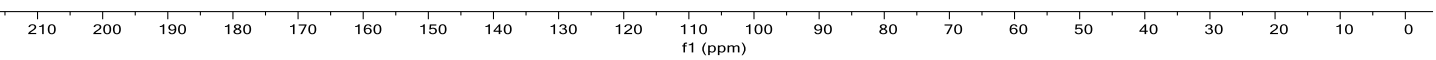




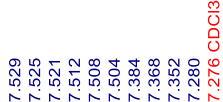
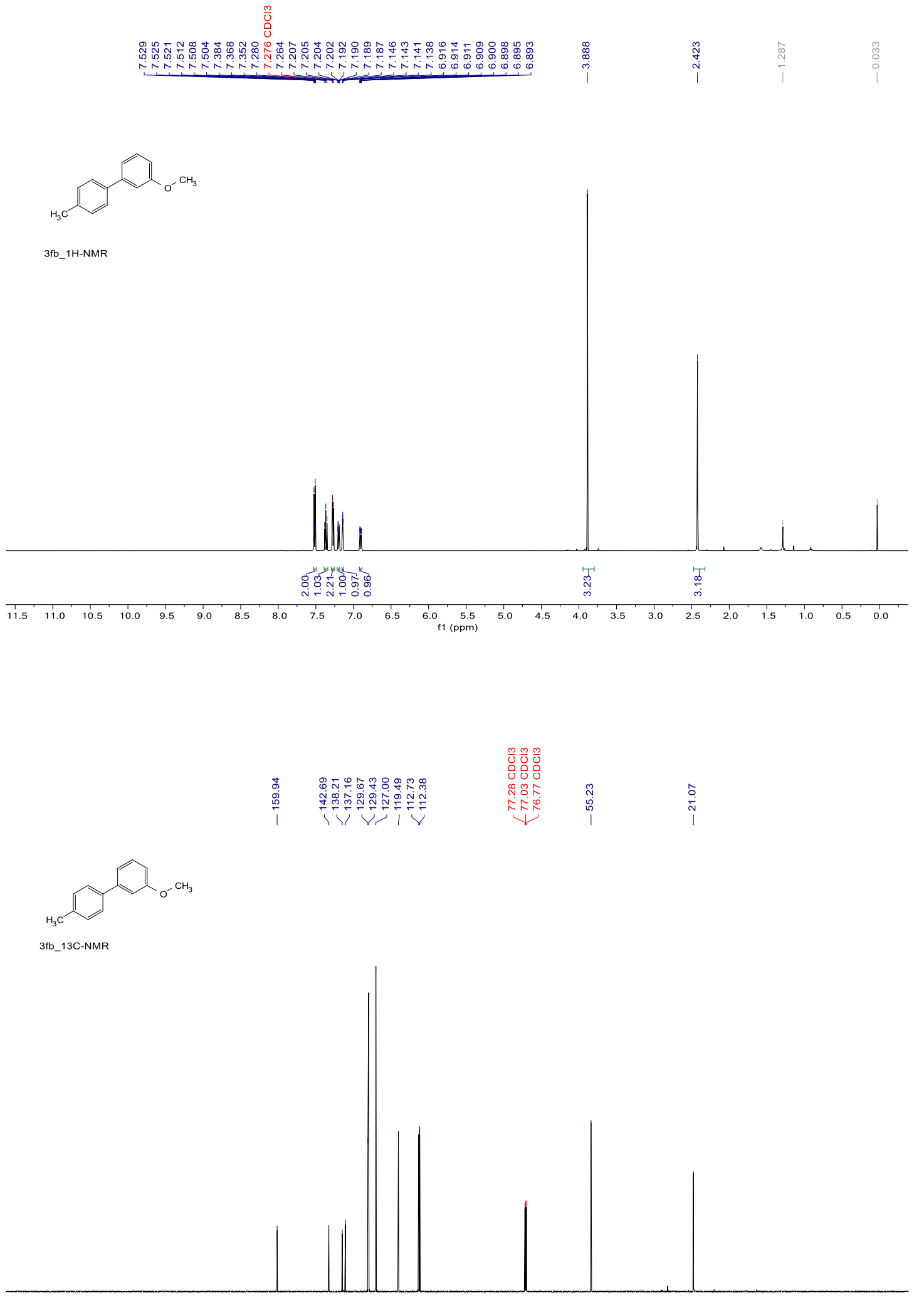

年

S17 


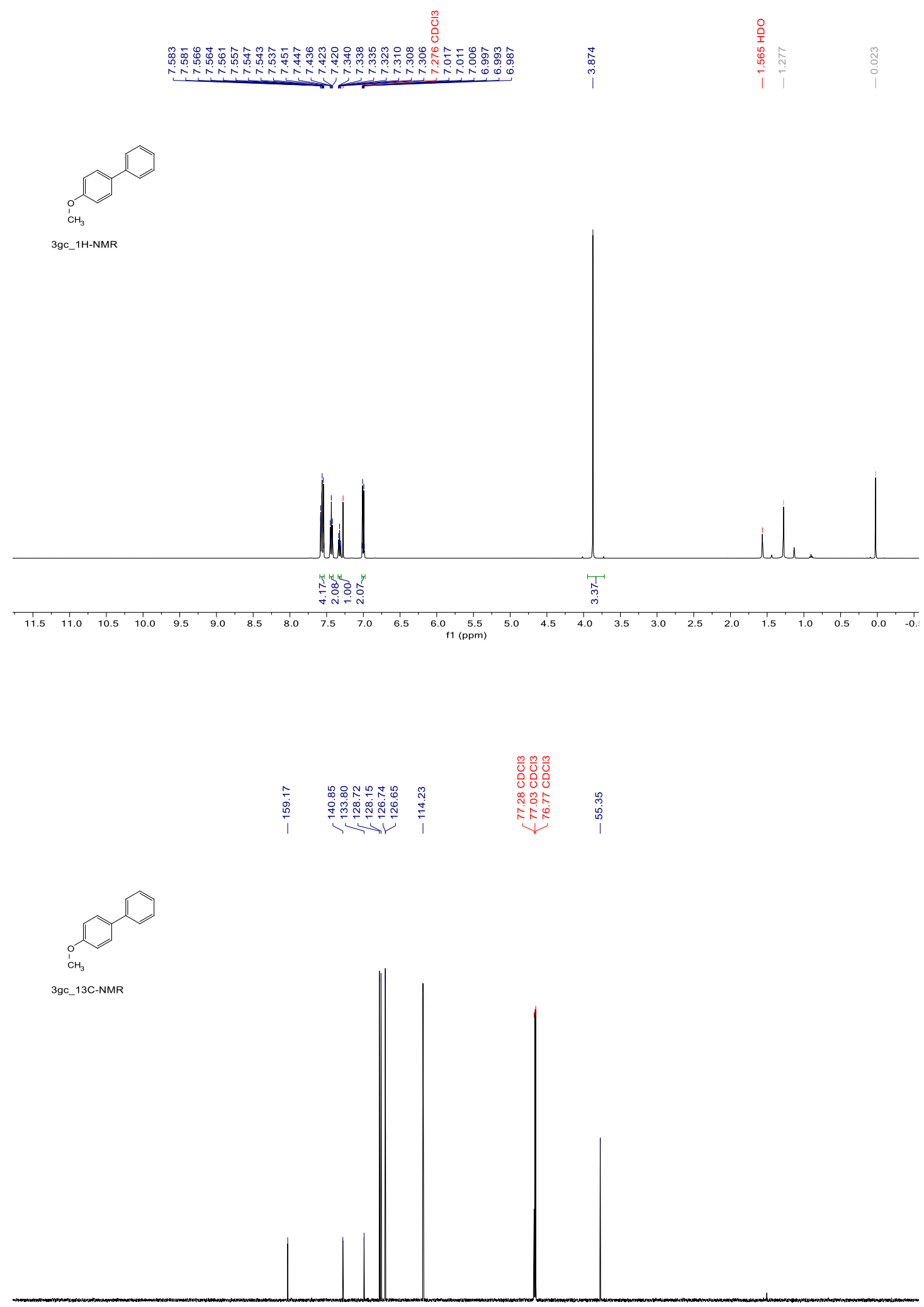

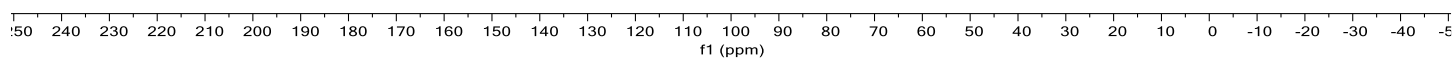




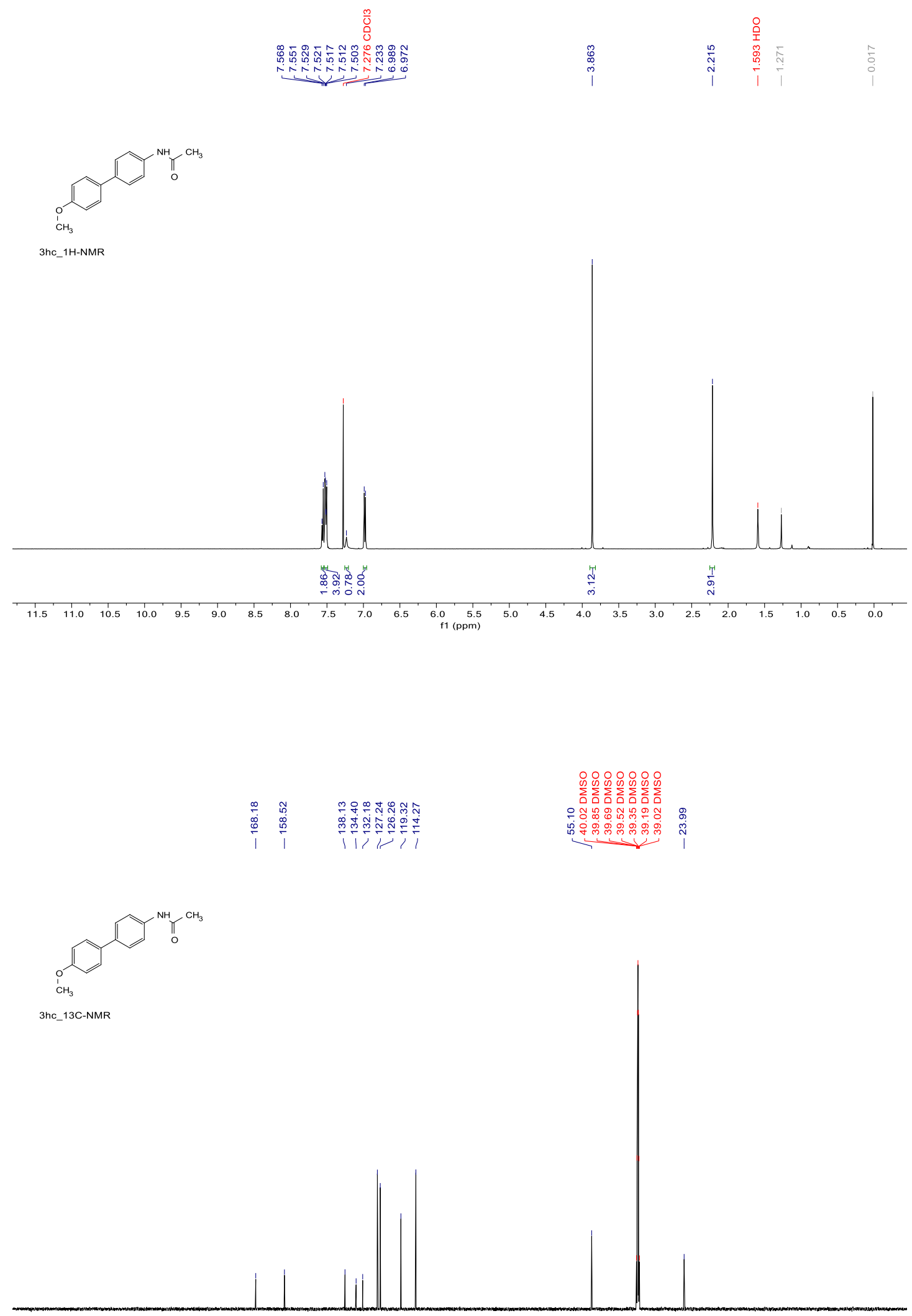

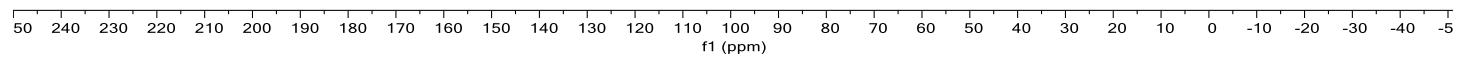




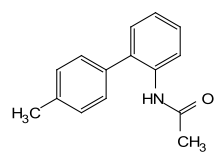

3ib_1 1 H-NMR
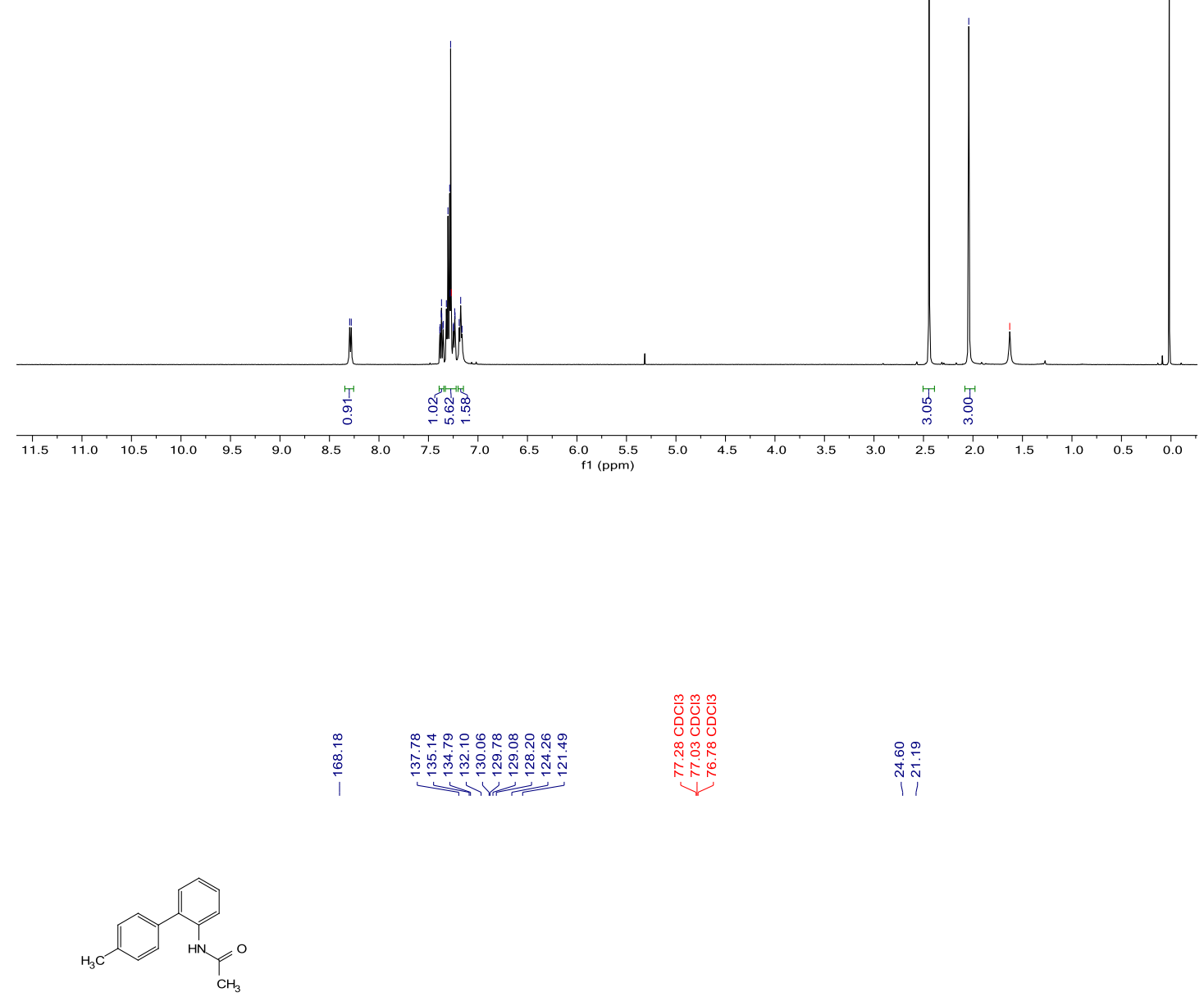

3ib_13C-NMR

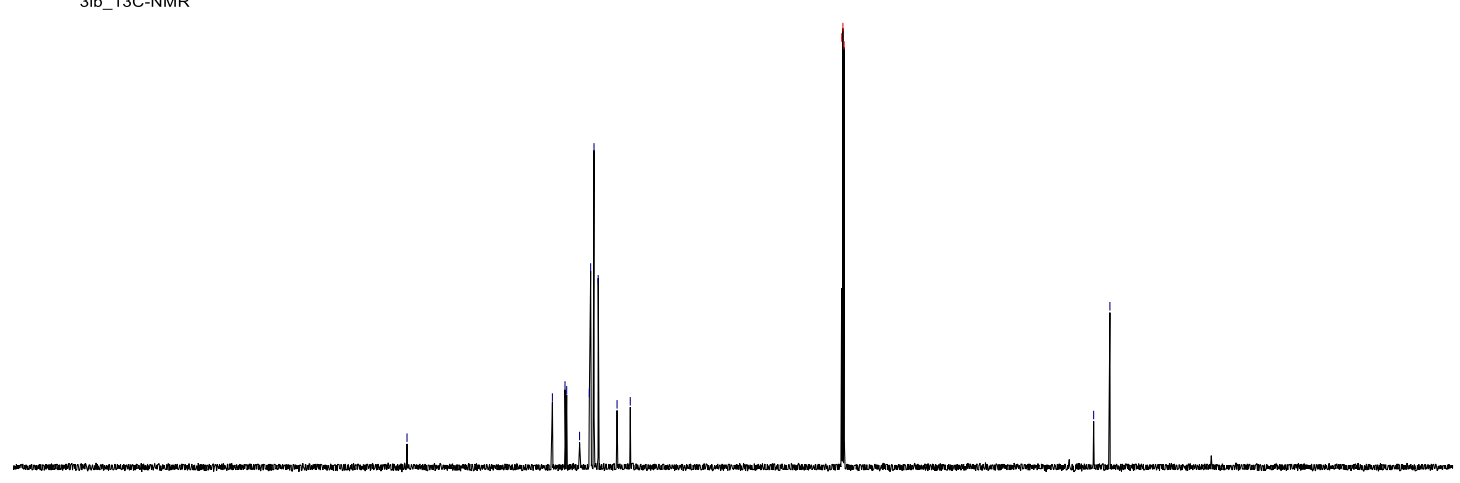

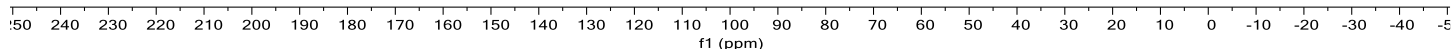



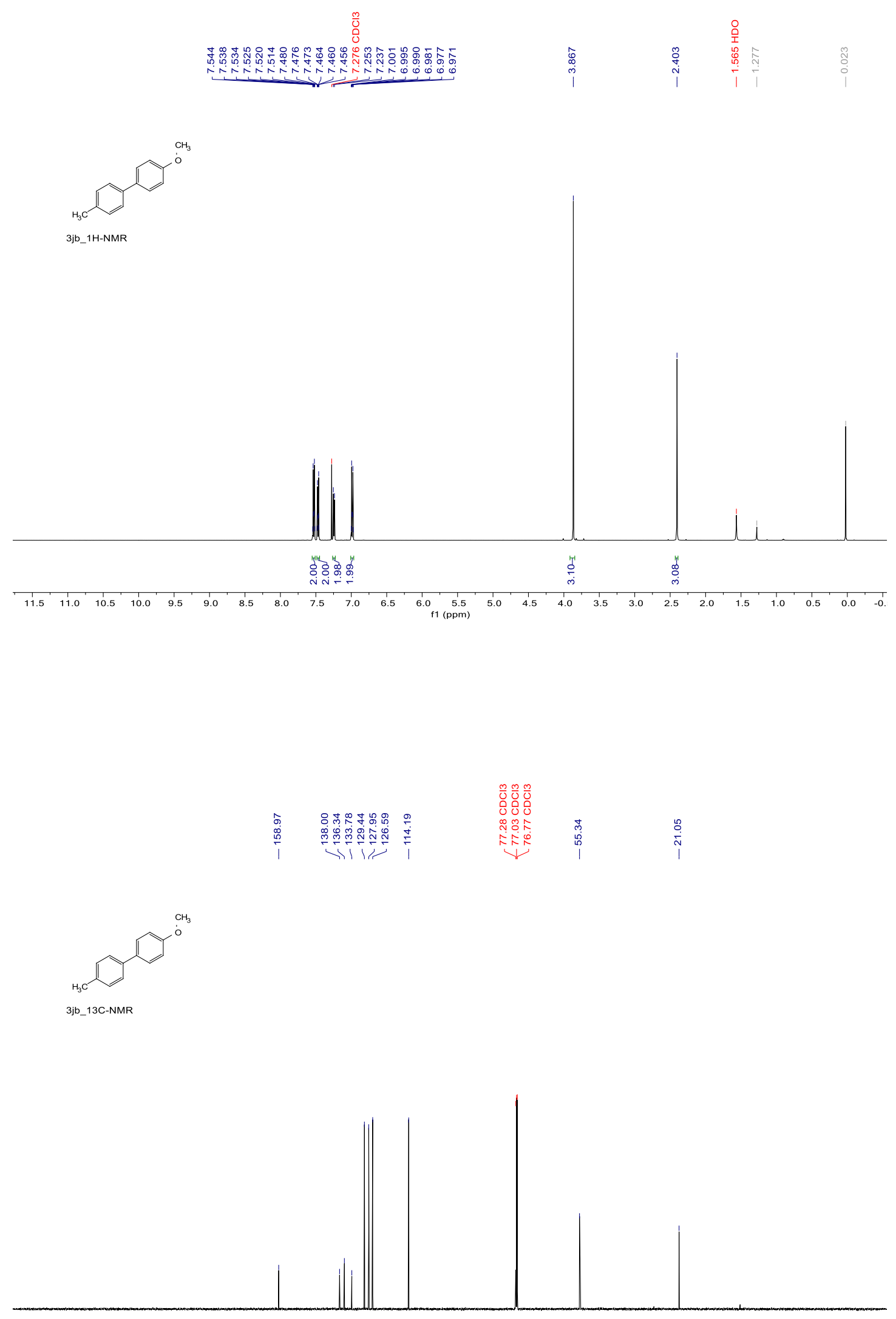

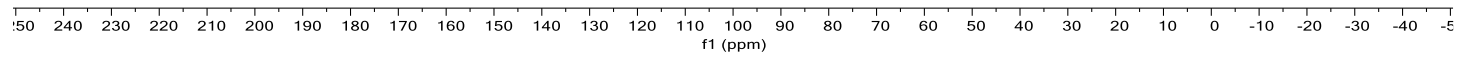




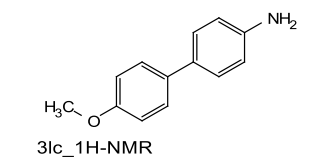

3lc_1 $1 \mathrm{H}-\mathrm{NMR}$
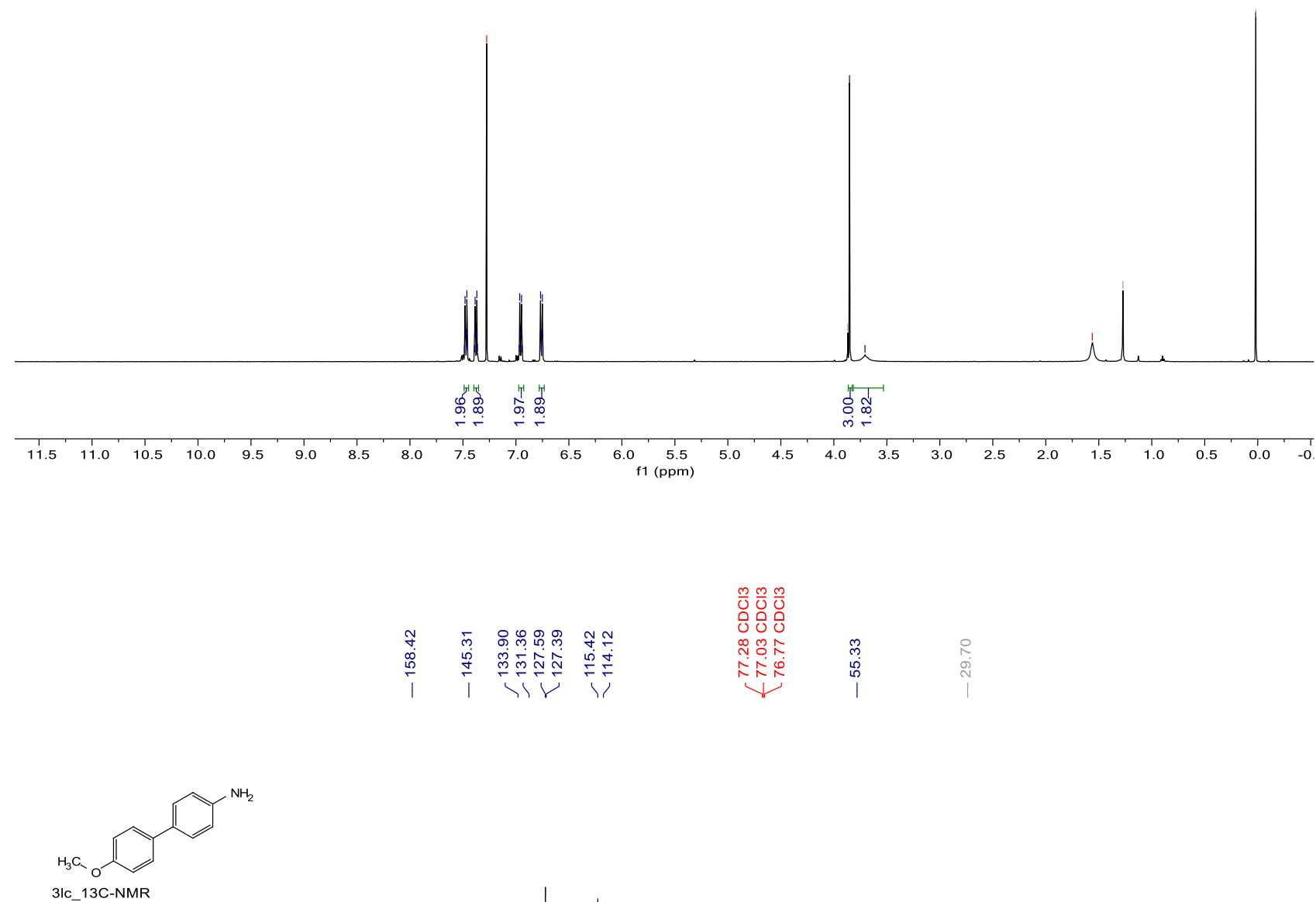

31c_13C-NMR

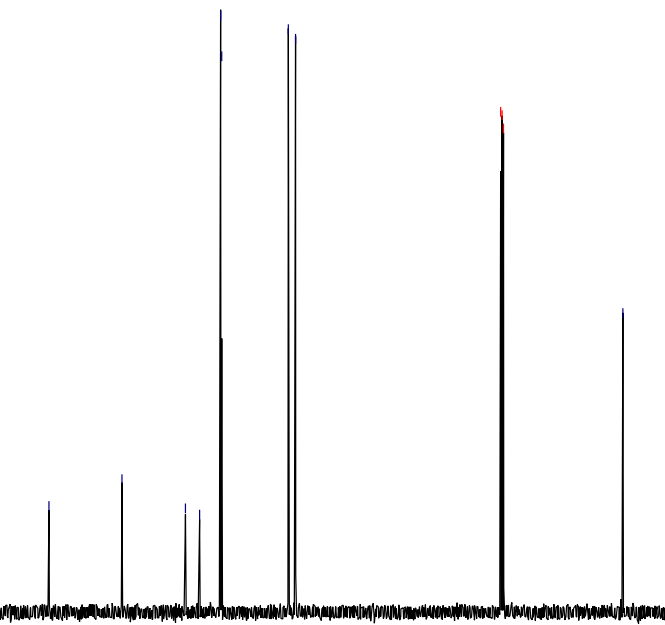

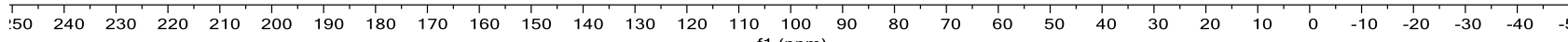


$\frac{0}{0}$

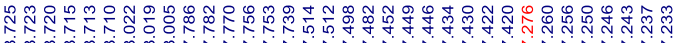

年

3ma_1H-NMR

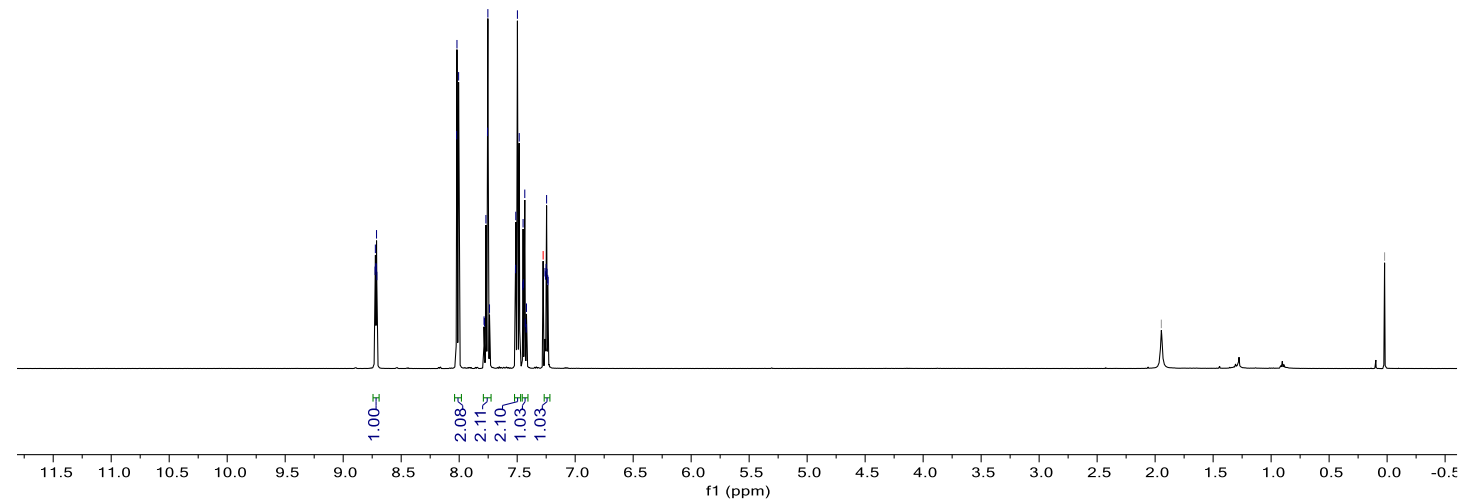

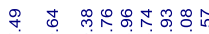

for

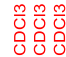

邹

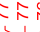

l l पारा

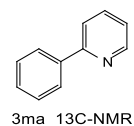

3ma_13C-NMR

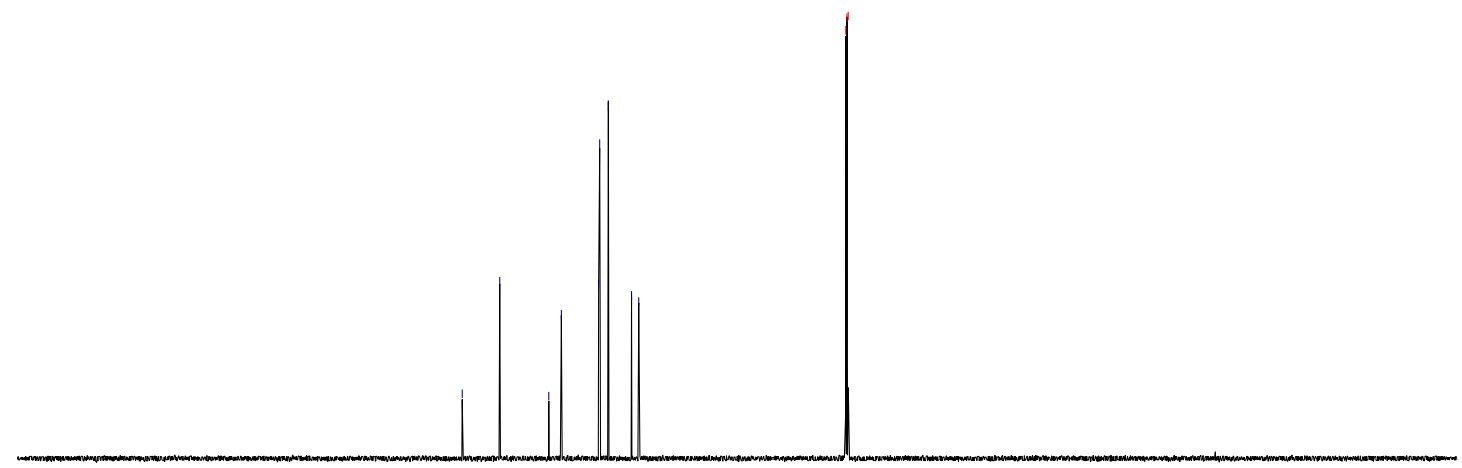

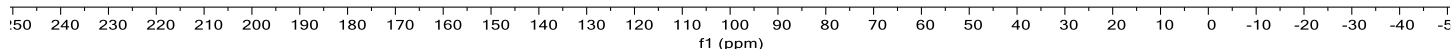



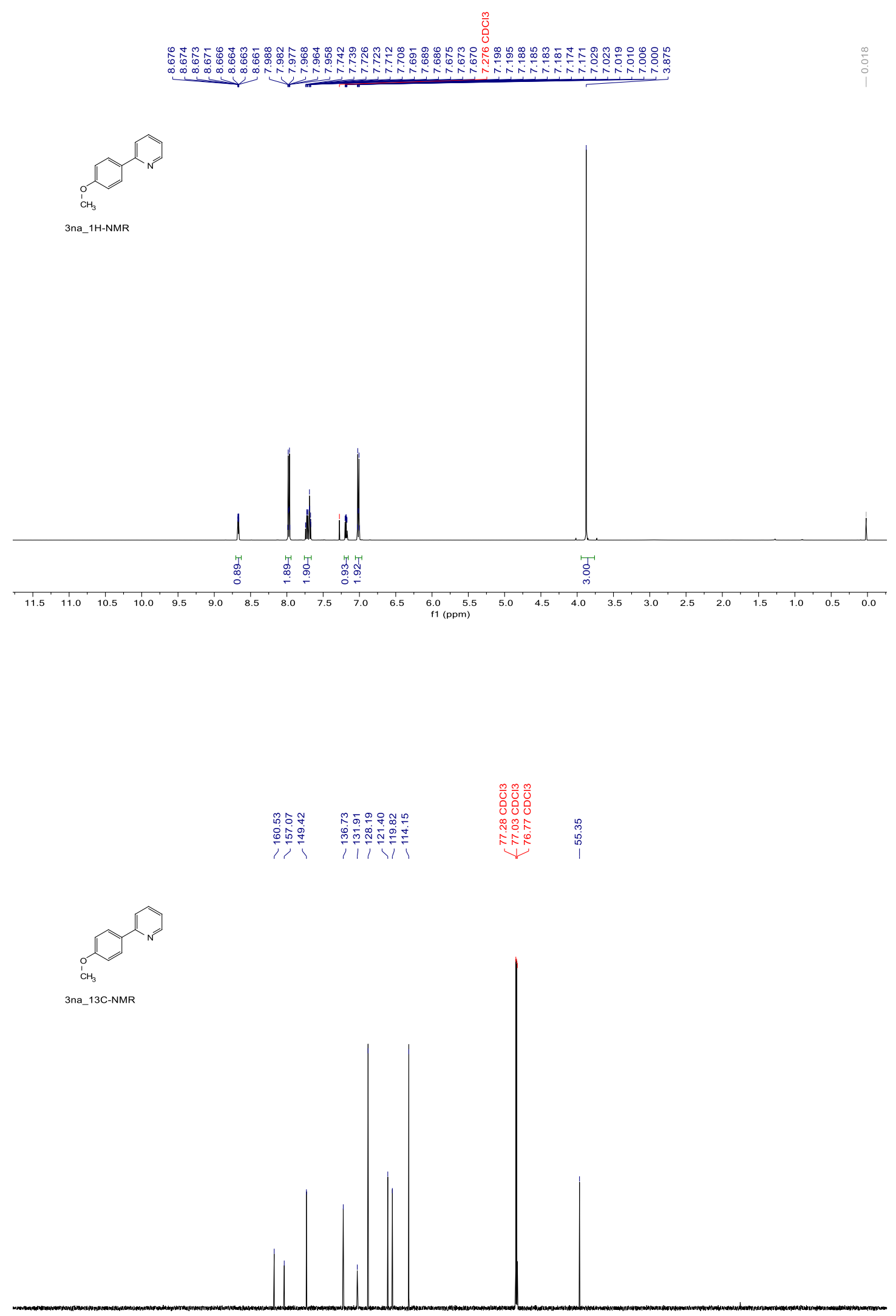

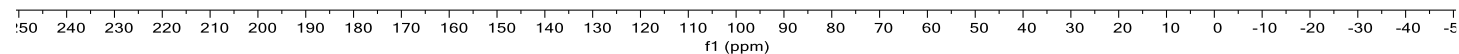



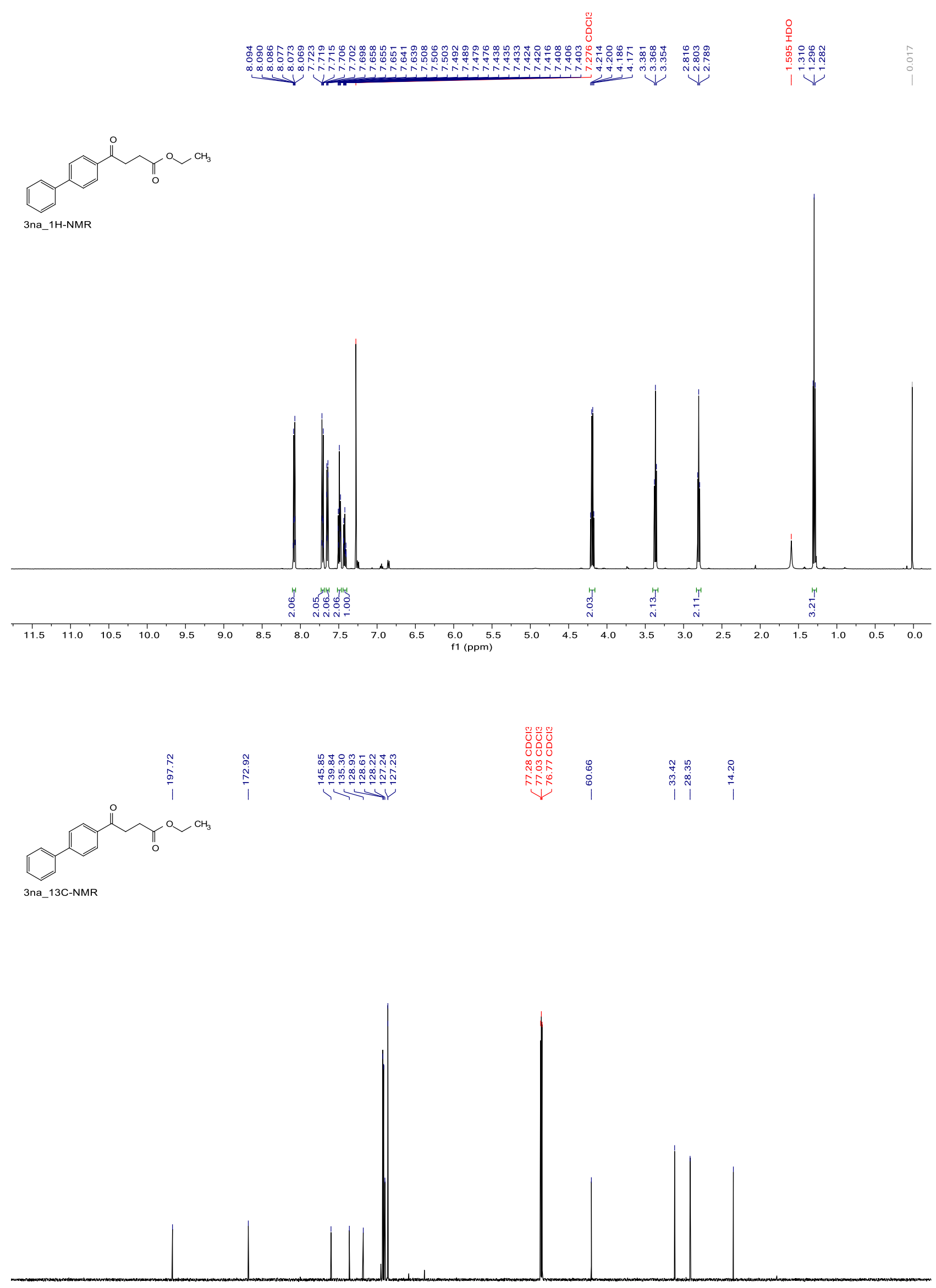

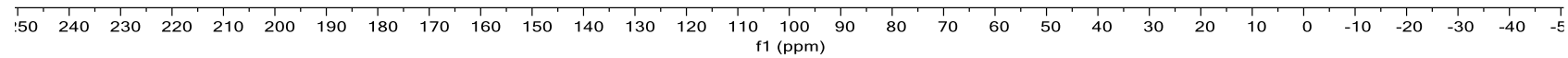

